\title{
Flora algal del río la Magdalena, Distrito Federal, MÉxico
}

\author{
Mónica Ramírez Vázquez, Yenny Beltrán Magos, Miriam Bojorge García, \\ Javier Carmona Jiménez, Enrique A. Cantoral Uriza, Francisco Valadez Cruz
}

Laboratorio de Ficología, Facultad de Ciencias, UNAM

A.P. 70-620. Ciudad Universitaria, Coyoacán, México, D.F.

Tel: 5256 224837. Fax: 5256 224828. e-mail: jcj@hp.fciencias.unam; eacu@hp.fciencias.unam.mx.

\begin{abstract}
Resumen. La flora algal de tres localidades del río La Magdalena, está constituida por 72 especies con las siguientes proporciones: 63 Bacillariophyceae, seis Cyanophyceae, una Chlorophyceae, una Ulvophyceae y una Charophyceae. Se describen las especies e ilustran con fotografías, se incluyen datos ambientales y referencias para México. Se encontraron 11 nuevos registros para la flora algal del país.

Palabras clave: algas, ríos de montaña, Ciudad de México.

Abstract. The result of the phycofloristic study of three places from La Magdalena's river were 72 algal species with the next proportions: 63 Bacillariophyceae, six Cyanophyceae, one Chlorophyceae, one Ulvophyceae and one Charophyceae. Is presented a brief description of the species, including environmental characteristics and photographs. Eleven new registers to the country are reported.
\end{abstract}

Key words: Algae, mountain streams, Mexico City.

L a flora algal de ambientes lóticos es abundante, diversa y juega un papel importante por ser productores primarios (Round, 1984). Diferentes autores mexicanos y extranjeros han trabajado ríos, manantiales y cascadas de San Luis Potosí, Tamaulipas, Hidalgo (Sheath y Cole, 1992; Montejano et al., 1997), Morelos (Valadez et al., 1996) y Puebla (Novelo, 1998), estos son estudios de regiones con características climáticas y geomorfológicas diferentes al Valle de México.

Las investigaciones sobre algas de ambientes lóticos en el Valle de México son escasas, actualmente existen numerosos manantiales y corrientes permanentes en la zona surponiente de la cuenca. Las primeras referencias datan del siglo pasado cuando Peñafiel (1884 En: Ortega, 1984) proporcionaron una lista de algas presentes en colectores de agua potable que circulaban en la ciudad. Transcurrieron 47 años antes de que apareciera el primer trabajo elaborado por investigadores nacionales (Sámano-Bishop y Sokoloff, 1931) y una serie de artículos donde se describían listados florísticos (Ortega, 1984). No obstante, la mayoría correspondían a cuerpos de agua lénticos o afluentes que ya desaparecieron o fueron entubados.

Como resultado de lo anterior y teniendo en cuenta que no existe hasta el momento una compilación que integre la flora algal de ríos del Valle de México, que son ambientes fundamentales para la presencia de las algas las cuales reflejan el estado de salud del cuerpo de agua, se desarrolló la presente investigación que pretende describir las especies en una primera aproximación a uno de los ríos más importantes del valle.

\section{Área de estudio}

El río La Magdalena es un afluente permanente que se ubica en la delegación Magdalena Contreras, D.F., en la demarcación del parque nacional. "Los Dinamos". Se localiza entre los $19^{\circ} 16^{\prime} 00^{\prime \prime} \mathrm{LN}$ y los $99^{\circ}$ 18' 22" LW (Geoposicionador GARMIN 45) con altitudes que varían de los 2,800 a los 3,170 msnm (Instituto Nacional de Estadística Geografía e Informática, 1995) (figura 1). Forma parte de la región hidrológica del río Pánuco, subcuenca lago Texcoco-Zumpango y su cauce corre a través de la Sierra del Ajusco 
en el cual se encuentran asentadas cuatro construcciones que en algún momento funcionaron como dinamos. El tercero y cuarto dinamo se encuentran en las localidades de Anamiquiac y Xalancocotla respectivamente. En el segundo dinamo se instaló una planta potabilizadora de agua, que desvía parte de ésta con el fin de abastecer a la comunidad; mientras que la otra continúa su recorrido hasta el siguiente dinamo y final del parque nacional. Una vez que entra a la zona urbana, continúa su recorrido hasta Santa Teresa y posteriormente se entuba, sale a superficie nuevamente a la altura de los viveros de Coyoacán entubándose definitivamente hasta descargar en la presa Anzaldo.

En la zona del parque se pueden reconocer dos periodos con diferente precipitación pluvial, uno de lluvias en los meses de junio a octubre y otro de secas que abarca los meses de noviembre a mayo. La temperatura del agua puede presentar un amplio gradiente que va de $\operatorname{los} 2^{\circ} \mathrm{C}$ en invierno a $12.8^{\circ} \mathrm{C}$ en primavera (tabla 1). En general el suelo de esta zona está compuesto de endosoles derivados de cenizas, de composición ácida y de textura ligera u ocasionalmente media o pesada. Además, el suelo puede estar mezclado con regosoles. El clima es semifrío subhúmedo con lluvias en verano $\mathrm{C}(\mathrm{E})\left(\mathrm{W}_{2}\right)$. La vegetación predominante es bosque de Pinus rudis, P. montezumae y Quercus rugosa, en suelos profundos y someros ocasionalmente muy pedregosos (Espinosa y Sarukhán, 1997).

\section{Material y método}

El río se colectó en siete ocasiones en las siguientes localidades: cuarto dinamo, segundo dinamo y camino a Santa Teresa, entre los meses de noviembre de 1997 a febrero de 1999. En el campo se colectaron los crecimientos algales visibles, tomando el fragmento de roca con cincel y martillo, o bien levantando la muestra con espátula. Cada crecimiento quedó referido a un número de muestra el cual incluyó el registro de los factores ambientales como: temperatura, $\mathrm{pH}$ y conductividad medidos con un potenciómetro Conductronic PC 18. El valor de los nutrientes se obtuvo con técnicas convencionales colorimétricas según $\mathrm{Hach}{ }^{\circledR}$ DR 2000. Los datos ambientales fueron definidos por el sistema propuesto por Lowe (1974) (tabla 1).

Las muestras se fijaron en formaldehido al $4 \%$ e incorporaron con las siglas MA a la sección ficológica del herbario FCME de la Facultad de Ciencias de la UNAM. Se realizaron preparaciones permanentes con gelatina glicerinada para la posterior observación de especies. Con este propósito se utilizaron microscopios compuestos con contraste de fase interdiferencial
(Reichert Zetopan) y para algunas Bacillariophyceae se utilizó microscopía electrónica de barrido (JEOL JSM-5310LV). Para la limpieza de diatomeas se empleó la técnica de oxidación ácida (Rushforth et al., 1984) y posteriormente se montaron en resina $\mathrm{Na}$ phrax® para su observación en microscopía de luz. Para apoyar la determinación de especies que requieren una observación a mayor aumento de caracteres se empleó microscopía electrónica; se colocó una gota de agua con diatomeas ya limpias con la técnica antes mencionada sobre cilindros que se dejaron secar a medio ambiente, $y$ posteriormente se cubrieron con oro-paladio.

Los siguientes trabajos fueron utilizados para la identificación de las Cyanophyceae: Desikachary (1959), Geitler (1930-1932), Anagnostidis y Komárek (1985, 1988), Komárek y Anagnostidis (1986, 1989, 1999), Gold-Morgan et al. (1994, 1996) y Montejano et al. (1993, 1997). Para las Bacillariophyceae: Germain (1981), Hustedt (1927-1930, 1930, 1931-1959), Krammer y Lange-Bertalot (1986, 1988, 1991a, 1991b) y Patrick y Reimer (1966, 1975), Transeau (1951), Ventakatamaran (1961) y Prescott et al. (1975) fueron usados para las Chlorophyceae; se siguó el esquema de clasificación propuesto por Hoek et al. 1995. Algunas especies quedaron sin determinar por la ausencia de estructuras reproductoras; no se incluyeron en la descripción de las especies.

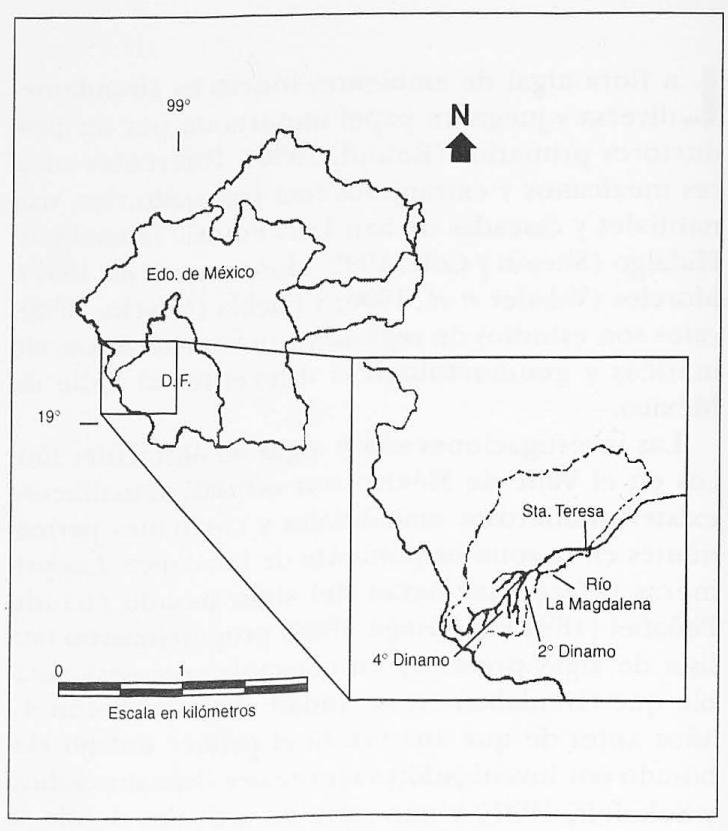

Figura 1. Área de estudio. 


\section{Resultados}

Se analizaron un total de 101 muestras y se determinaron un total de 72 taxa, de las cuales $6(8.3 \%)$ pertenecen a Cyanophyceae, $1(1.4 \%)$ a Chlorophyceae, $1(1.4 \%)$ a Ulvophyceae, $1(1.4 \%)$ a Charophyceae y $63(87.5 \%)$ a las Bacillariophyceae. Se presentan $11(15.3 \%)$ nuevos registros para el país (*).

Se observaron 9 géneros que forman crecimientos visibles que no se incorporan en las descripciones por no llegar a especie por falta de caracteres para este nivel, que fueron: Spirogyra, Mougeotia, Zygnema (Charophyceae), Chlorococcum, Oedogonium, Ulothrix (Chlorophyceae), Nostoc, Scytonema (Cyanophyceae) y Vaucheria (Xanthophyceae).

Las localidades con mayor número de especies comunes fueron el cuarto y segundo dinamo con 32 y el cuarto dinamo y camino a Santa Teresa con seis; mientras que camino a Santa Teresa y segundo dinamo no presentaron especies en común. Las especies que se presentaron en las tres localidades, y en casi todas las fechas de colecta fueron 14 Bacillariophyceae; y las especies distribuidas en una sola localidad fueron una para el camino a Santa Teresa, cinco para el segundo dinamo y 24 en el cuarto dinamo (tabla 2).

Se colectaron 45 especies presentes en ambas épocas del año (secas-lluvias); 29 resultaron exclusivas de la época de secas y siete de la época de lluvias. Los microambientes donde se registraron fueron: 49 en zona de deslizamiento, 57 en remanso, cinco subaéreas y una en estanque. Las formas de vida: 56 perifíticas, 46 epilíticas, 15 epífitas, 11 epipélicas, dos planctónicas y tres sobre plástico.
Clase Bacillariophyceae

Orden Centrales

Suborden Coscinodiscineae

Familia Thalassiosiraceae

Aulacoseira granulata (Ehrenberg) Simonsen 1979 (figuras 1-2)

Largo 4.5-20.3 $\mu \mathrm{m}$; ancho 3.6-6.3 $\mu \mathrm{m}$; estrías $11-15 /$ $10 \mu \mathrm{m}$.

Datos ecológicos: planctónica, deslizamiento, remanso. Número de muestra: MA 37, 57, 64, 90, 91, 95.

Referencias para México: Morelos (Valadez, 1998); Estado de México (Caballero y Ortega, 1998); Puebla (Novelo, 1998); Guanajuato (García, 1997); Guanajuato y Michoacán (Metcalfe y O’hara, 1992).

Cyclotella meneghiniana Kützing 1844 (figura 3)

Diámetro 10.3-14.6 $\mu \mathrm{m}$; estrías 8-10/10 $\mu \mathrm{m}$.

Datos ecológicos: planctónica, remanso.

Número de muestra: MA 30, 51, 55, 57.

Referencias para México: Estado de México (Caballero et al., 1997); Michoacán (Hutchinson et al., 1956); Valle de México (Flores-Granados, 1980); Hidalgo (Ortega, 1984; Chang, 1989); Yucatán (Sánchez, 1985); México central (Metcalfe, 1985, 1988; Metcalfe et al., 1991; Metcalfe, 1992-1993); Guanajuato y Michoacán (Metcalfe y O’hara, 1992); San Luis Potosí (Cantoral-Uriza, 1990, 1993, 1997; Cantoral-Uriza y Montejano-Zurita, 1993; Cantoral-Uriza et al., 1997); Morelos (Valadez, 1992, 1998; Valadez et al., 1996); Puebla (Cuesta, 1993; Novelo, 1998); Guanajuato (García, 1997).

Tabla 1. Características físicoquímicas de los sitios de colecta en el río La Magdalena.

\begin{tabular}{|c|c|c|c|}
\hline Variables/Localidad & 4o. Dinamo & 20. Dinamo & Camino Sta. Teresa \\
\hline $\begin{array}{l}\text { Fecha de colecta y relación } \\
\text { del número de muestra }\end{array}$ & $\begin{array}{r}\text { 23-XI-97 (MA1-23) } \\
8-11-98 \text { (MA24-47) } \\
\text { 6-VII-98(MA55-60) } \\
\text { 30-VIII-98(MA65-70 } \\
\text { 19-XII-98 (MA74-88) } \\
24-I-99 \text { (MA95-101) }\end{array}$ & $\begin{array}{r}8-11-98 \text { (MA48-54) } \\
6-V I I-98 \text { (MA61-64) } \\
28-X-98 \text { (MA71-73) } \\
24-1-99 \text { (MA92-94) }\end{array}$ & 24-I-99 (MA89-91) \\
\hline Temperatura $\left({ }^{\circ} \mathrm{C}\right)$ & $2.0-11.0$ & $6.0-12.8$ & 4.7 \\
\hline $\mathrm{pH}$ & $6.3-7.7$ & $7.3-7.8$ & 6.8 \\
\hline Conductividad $(\mu S)$ & $39.4-102.0$ & $50.3-90.1$ & 59.1 \\
\hline Fosfatos $\mathrm{mg} \mathrm{l}^{-1}\left(\mathrm{PO}_{4}\right)$ & - & 0.2 & 2.19 \\
\hline Sulfatos $\mathrm{mg} \mathrm{l}^{-1}\left(\mathrm{SO}_{4}\right)$ & - & 11.0 & 44.0 \\
\hline Nitratos mg $\left.\right|^{-1}\left(\mathrm{NO}_{3}^{4}-\mathrm{N}\right)$ & - & 0.7 & 6.2 \\
\hline Alcalinidad $\mathrm{mgl}^{-1}\left(\mathrm{CaCO}_{3}\right)$ & - & 3.72 & 3.16 \\
\hline Dureza-Ca mg |-1 $\left(\mathrm{CaCO}_{3}\right)$ & - & 0.01 & 0.01 \\
\hline Dureza-Mg mgl-1 $\left(\mathrm{CaCO}_{3}\right)$ & - & 0.90 & 0.76 \\
\hline Silicatos $\mathrm{mg} \mathrm{l}^{-1}\left(\mathrm{SiO}_{3}\right)$ & - & 32.1 & 50.5 \\
\hline
\end{tabular}


Mónica Ramírez Väzuez, et al.

Tabla 2. Lista de especies y su distribución en las localidades de estudio.

Géneros/especies $\quad 4^{\circ}$ Dinamo $\quad 2^{\circ}$ Dinamo Camino Sta. Teresa

\section{BACILLARIOPHYCEAE}

Achnanthes hungarica

Achnanthes inflata

Achnanthes lanceolata ssp.

lanceolata var. lanceolata

Achnanthes minutissima

var. minutissima

Amphora commutata

Aulacoseira granulata

Berkella linearis

Caloneis bacillum

Caloneis silicula

Cocconeis placentula

var. lineata

Cyclotella meneghiniana

Cymbella amphicephala

Cymbella leptoceros

Cymbella mexicana

Cymbella naviculiformis

Cymbella norvegica

Cymbella silesiaca

Cymbella sinuata

Diatoma mesodon

Diploneis elliptica

Diploneis oblongella

Epithemia turgida

var. granulata

Eunotia exigua

Fragilaria bidens

Fragilaria construens

var. construens

Fragilaria pinnata

Fragilaria ulna

Gomphonema acuminatum

Gomphonema grovei

Gomphonema parvulum

Gomphonema truncatum

Hantzschia amphioxys

Melosira varians

Meridion circulare

var. constrictum

Navicula charlatii

Navicula cryptocephala

Navicula cuspidata

Navicula mutica

Navicula nivalis

Navicula pseudocutiformis

Navicula radiosa

Navicula reinhardtii

Navicula subrhynchocephala

Neidium iridis

Nitzschia acicularis

Nitzschia amphibia

$x$

X

X

$X$

$x$

$X$

$X$

X

X

$X$

$x$

$x$

$x$

$x$

$X$

$x$

$x$

$x$

$X$

X

$X$

$x$

$x$

X

$x$

$x$

$x$

$x$

X

X

X

$X$

$X$

$x$

$X$

$x$

$x$

$X$

$x$

$x$

X

$x$

$X$
X

X

X

$x$

X

$x$

$x$

X

$x$
$x$

$x$

$x$

X

X

$x$

$x$

$x$

$X$

X

$x$

X

X

$x$

X

X

X

$x$

X

$x$

X

X 
Tabla 2. Continúa

\begin{tabular}{|c|c|c|c|}
\hline Céneros/especies & $4^{\circ}$ Dinamo & $2^{\circ}$ Dinamo & Camino Sta. Teresa \\
\hline Nitzschia clausii & $x$ & $\mathrm{x}$ & \\
\hline Nitzschia heufleriana & $x$ & $x$ & \\
\hline Nitzschia palea & $x$ & $\mathrm{x}$ & $x$ \\
\hline Orthoseira roeseana & $x$ & $x$ & $x$ \\
\hline Pinnularia borealis & $x$ & $\mathrm{x}$ & \\
\hline Pinnularia microstauron & $x$ & $x$ & \\
\hline Pinnularia simmilis & $x$ & $x$ & \\
\hline Pinnularia viridis & $x$ & $x$ & \\
\hline Rhoicosphenia abbreviata & $x$ & $x$ & \\
\hline Rhopalodia gibba & $\mathrm{x}$ & & $\mathrm{x}$ \\
\hline Rhopalodia gibberula & $x$ & $\mathrm{x}$ & \\
\hline Stauroneis phoenicenteron & $x$ & $x$ & \\
\hline Stephanodiscus rotula & $\mathrm{x}$ & & $x$ \\
\hline Surirella angusta & $x$ & & \\
\hline Surirella bifrons & $x$ & & \\
\hline Surirella brightwellii & $x$ & & \\
\hline Surirella tenera & $x$ & $x$ & \\
\hline \multicolumn{4}{|l|}{ CYANOPHYCEAE } \\
\hline Chamesiphon cf. confervicolus & $x$ & & \\
\hline Mantellum sp. & & $\mathrm{x}$ & \\
\hline Myxosarcina sp. & & $x$ & \\
\hline Phormidium retzii & $x$ & & \\
\hline Oscillatoria limosa & $x$ & & \\
\hline Xenococcus cf. willei & $x$ & $x$ & \\
\hline \multicolumn{4}{|l|}{ CHLOROPHYCEAE } \\
\hline Scenedesmus armatus & $x$ & & \\
\hline \multicolumn{4}{|l|}{ ULVOPHYCEAE } \\
\hline Prasiola mexicana & $x$ & $x$ & \\
\hline \multicolumn{4}{|l|}{ CHAROPHYCEAE } \\
\hline Penium spinospermum & $x$ & & \\
\hline
\end{tabular}

Orthoseira roeseana (Rabenhorst) O`Meara 1876 (figuras 4-5)

Diámetro 23.8-32.5 $\mu \mathrm{m}$; estrías $16 / 10 \mu \mathrm{m}$; vista cingular, largo $32.9 \mu \mathrm{m}$; ancho $13.88 \mu \mathrm{m}$.

Datos ecológicos: perifítica, epilítica, remanso.

Número de muestra: MA 93, 95.

Referencias para México: Oaxaca (Tavera et al., 1994)

* Stephanodiscus rotula (Kützing) Hendè 1964 (figura 6)

Células cilíndricas, superficie valvar ligeramente ondulada; estrías radiales evidentes en los margenes; área central con estriación radial e irregular. Diámetro 915.5; estrías 20-26 / $10 \mu \mathrm{m}$; espinas 8-13 $\mu \mathrm{m}$.

Datos ecológicos: epilítica, deslizamiento.

Número de muestra: MA 15, 37, 38.

Referencias para México: Primer registro.

\section{Familia Melosiraceae}

Melosira varians Agardh 1827 (figura 7)

Largo 12.7-13.7 $\mu \mathrm{m}$; ancho 13.1-14.4 $\mu \mathrm{m}$.

Datos ecológicos: perifítica, epilítica, deslizamiento, remanso. 


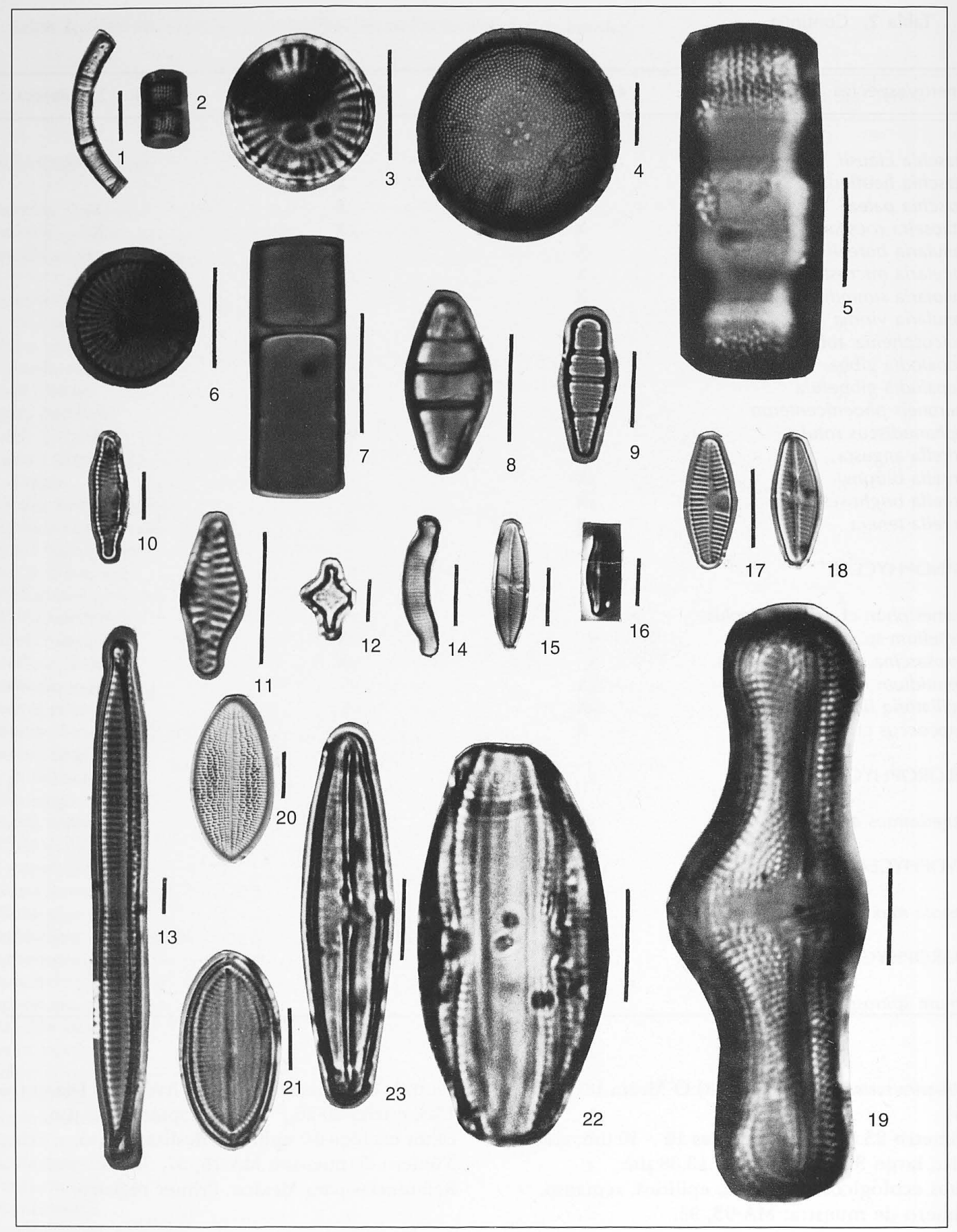

Figura 2. 1-2. Aulacoseira granulata; 3. Cyclotella meneghiniana; 4-5. Orthoseira roeseana; 6. Stephanodiscus rotula; 7. Melosira varians; 8. Diatoma mesodon; 9. Meridion circulare var. constrictum; 10. Fragilaria bidens; 11. F. pinnata; 12. F. construens; 13. F. ulna; 14. Eunotia exigua; 15. Achnanthes hungarica; 16. A. minutissima var. minutissima; 17-18. A. lanceolata spp. lanceolata var. lanceolata; 19. A. inflata; 20-21. Cocconeis placentula var. lineata; 22. Amphora commutata; 23. Berkella linearis. Escala de la barra $10 \mu \mathrm{m}$. 
Número de muestra: MA $15,25,55,57,58,64,88$, 90, 93.

Referencias para México: Estado de México (Bradbury, 1971); México central (Kusel-Fetzmann, 1973); Hidalgo (Ortega, 1984); Puebla (Novelo, 1998).

Orden Pennales

Suborden Araphidineae

Familia Fragilariaceae

*Diatoma mesodon (Ehrenberg) Kützing 1844 (figura 8) Valva lanceolada con extremos redondeados; isopolar; en vista valvar con cuatro cámaras. Largo 12.119-9 $\mu \mathrm{m}$; ancho 5.9-8.8 $\mu \mathrm{m}$; estrías muy finas dispuestas paralelamente a los engrosamientos de la pared celular:

Datos ecológicos: perifítica, epilítica, epífita, remanso.

Número de muestra: MA 30, 38, 49, 55, 57, 58, 63, 64, 90, 93.

Referencias para México: Primer registro.

* Meridion circulare (Greville) C.A. Agardh 1831 var. constrictum (Ralfs) Van Heurck 1880 (figura 9)

Valva heteropolar, con extremos redondeados. Largo 16-22.1 $\mu \mathrm{m}$; ancho 4.6-5.1 $\mu \mathrm{m}$; estrías 22-24 / 10 $\mu \mathrm{m}$; costillas 3-4 / $10 \mu \mathrm{m}$.

Datos ecológicos: perifítica, epilítica, deslizamiento, remanso.

Número de muestra: MA 15, 55, 91.

Referencias para México: Primer registro.

\section{* Fragilaria bidens Heiberg 1863 (figura 10)}

Valva recta, con una convexidad en ambos lados en la región media, extremos ligeramente rostrado capitados; desfazamiento de estrías en la zona central. Largo 17.1-30.6 $\mu \mathrm{m}$; ancho 4.1-5.3 $\mu \mathrm{m}$; estrías alternas 10-12/ $10 \mu \mathrm{m}$.

Datos ecológicos: perifítica, epilítica, deslizamiento, remanso.

Número de muestra: MA 30, 38, 49, 51, 55, 57, 58, 61, 63, 64, 66, 90, 93.

Referencias para México: Primer registro.

Fragilaria construens (Ehrenberg) Grunow 1862 var: construens (figura 12)

Largo $18.8 \mu \mathrm{m}$; ancho $7.1 \mu \mathrm{m}$; estrías $18-20 / 10 \mu \mathrm{m}$. Datos ecológicos: epipélica, epífita, remanso.

Número de muestra: MA 37, 49.

Referencias para México: Hidalgo (Ortega, 1984); México central (Metcalfe, 1988; Metcalfe et al., 1991); Morelos (Valadez, 1998); Estado de México (Sugiura et al. 1994); Guanajuato (García, 1997); San Luis Potosí (Cantoral-Uriza, 1997); Puebla (Novelo, 1998).
Fragilaria pinnata Ehrenberg 1843 (figura 11) Largo 6.2-11.2 $\mu \mathrm{m}$; ancho 3.6-6.3 $\mu \mathrm{m}$; estrías 10-11/ $10 \mu \mathrm{m}$.

Datos ecológicos: perifítica, deslizamiento.

Número de muestra: MA 37.

Referencias para México: Morelos (Valadez, 1998); Estado de México (Kusel-Fetzman, 1973; Bradbury, 1971); México Central (Metcalfe, 1988).

Fragilaria ulna (Nitzsch) Lange-Bertalot 1980 (figura 13) Largo 122.4-153.9 $\mu \mathrm{m}$; ancho 10.2-15.5 $\mu \mathrm{m}$; estrías paralelas y punteadas $5-6 / 10 \mu \mathrm{m}$.

Datos ecológicos: perifítica, epilítica, deslizamiento, remanso.

Número de muestra: MA 25, 30, 34, 38, 42, 49, 51 , $55,57,58,61,63,64,66,93,95$.

Referencias para México: Valle de México (FloresGranados, 1980); Oaxaca (Figueroa, 1984; Tavera y Gonzalez-Gonzalez, 1990; Tavera et al., 1994); Hidalgo (Ortega, 1984; Chang, 1989); Puebla (Avila, 1985, 1989; Navarro, 1988; Ibarra, 1992; Cuesta, 1993; Ibarra y Novelo, 1997; Novelo 1998); México central (Kusel-Fetzmann, 1973; Metcalfe, 1985, 1988); San Luis Potosí (Cantoral-Uriza, 1990, 1993, 1997; CantoralUriza y Montejano-Zurita, 1993; Cantoral-Uriza et al., 1997); Yucatán (Sánchez, 1985).

Suborden Raphidineae

Familia Eunotiaceae

* Eunotia exigua (Brébisson ex Kützing) Rabenhorst 1864 (figura 14)

Valva ligeramente arqueada con extremos redondeados; estrías finamente punteadas, paralelas en el centro y radiadas en los polos. Largo 19-20.1 $\mu \mathrm{m}$; ancho 2.9$3.7 \mu \mathrm{m}$; estrías $18-20 / 10 \mu \mathrm{m}$.

Datos ecológicos: epífita, deslizamiento, remanso.

Número de muestra: MA 30, 77, 80, 90.

Referencias para México: primer registro.

\section{Familia Achnanthaceae}

Achnanthes hungarica (Grunow) Grunow in Cleve \& Grunow 1880 (figuras 15)

Largo $25.6 \mu \mathrm{m}$; ancho $6.1 \mu \mathrm{m}$; estrías $23 / 10 \mu \mathrm{m}$.

Datos ecológicos: epilítica, deslizamiento, remanso.

Número de muestra: MA 90.

Referencias para México: México central (Metcalfe, 1985,1988; Metcalfe et al., 1991; Metcalfe, 1992-1993); Estado de México (Bradbury, 1971).

Achnanthes inflata (Kützing) Grunow in Cleve \& Grunow 1880 var: inflata (figura 19)

Largo $58.7 \mu \mathrm{m}$, ancho $18.7 \mu \mathrm{m}$; estrías $12 / 10 \mu \mathrm{m}$. 


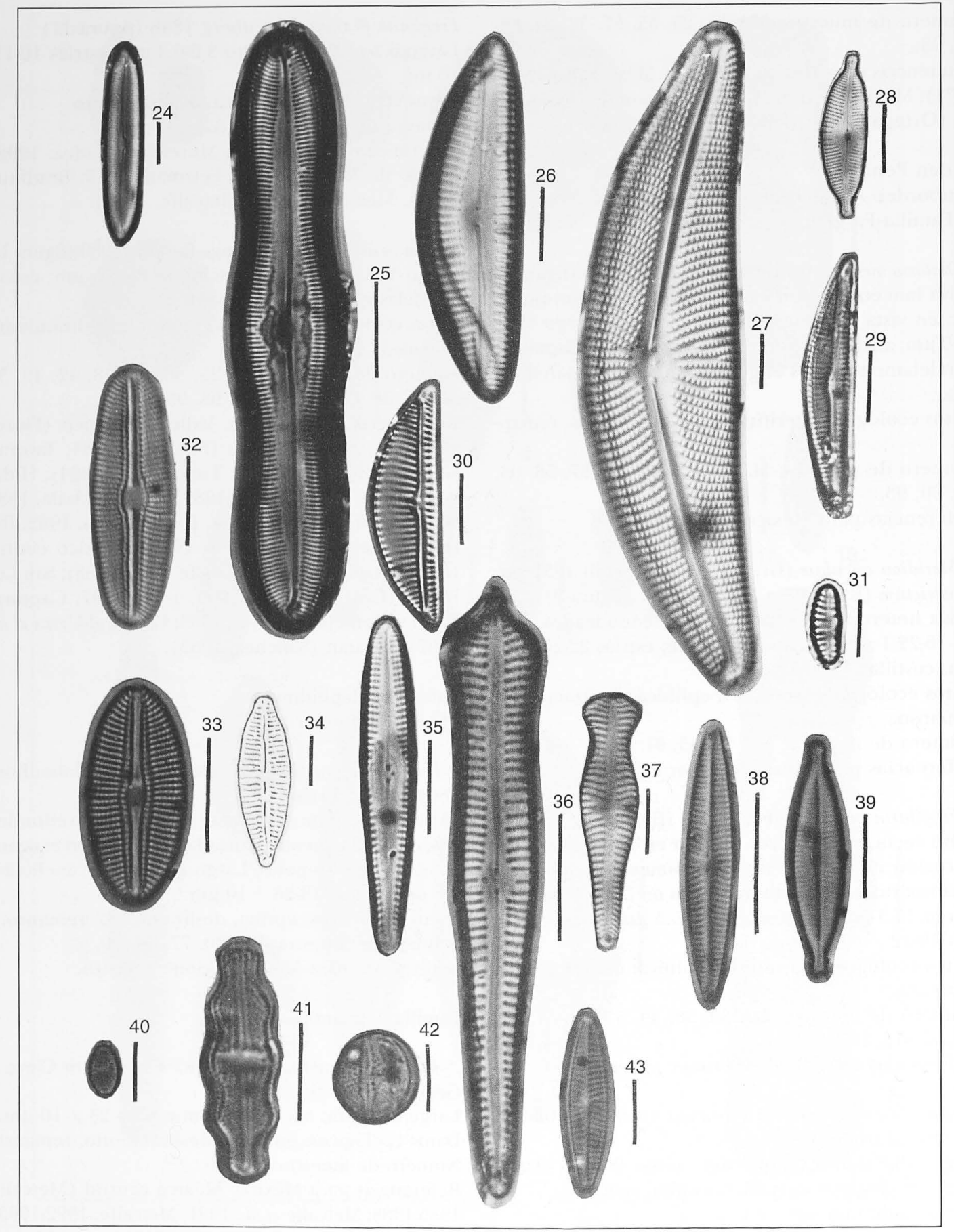

Figura 3. 24. Caloneis bacillum; 25. Caloneis silicula; 26. Cymbella leptoceros; 27. C. mexicana; 28. C. naviculiformis; 29. C. norvegica; 30. C. silesiaca; 31. C. sinuata; 32. Diploneis oblongella; 33. D. elliptica; 34. Gomphonema parvulum; 35. G. grovei; 36. G. acuminatum; 37. G. truncatum; 38. Navicula radiosa; 39. N. subrhynchocephala; 40. N. mutica; 41. N. nivalis; 42. N. pseudoscutiformis; 43. N. cryptocephala. Escala de la barra $10 \mu \mathrm{m}$. 
Datos ecológicos: epipélica, remanso.

Número de muestra: MA 93.

Referencias para México: Puebla (Avila, 1989; Cuesta, 1993; Novelo, 1998); San Luis Potosí (Carmona 1993; Cantoral-Uriza, 1997; Cantoral-Uriza et al., 1997); Oaxaca (Tavera et al., 1994).

Achnanthes lanceolata (Brébisson) Grunow in Cleve \& Grunow 1880 ssp. lanceolata var. lanceolata (figuras 1718)

Largo 11.3-20.1 $\mu \mathrm{m}$; ancho 4.8-6.7 $\mu \mathrm{m}$; estrías radiales y gruesas, centrales $13-15 / 10 \mu \mathrm{m}$ y terminales $11-13 / 10 \mu \mathrm{m}$.

Datos ecológicos: perifítica, epilítica, deslizamiento, remanso.

Número de muestra: MA 25, 30, 34, 38, 42, 49, 51, $55,57,58,61,64,66,77,88,91,93,95$.

Referencias para México: México central (Kusel-Fetzmann, 1973; Metcalfe, 1985, 1988); Oaxaca (Tavera y González-González, 1990; Tavera et al., 1994); Morelos (Valadez, 1992, 1998); Puebla (Cuesta, 1993; Ibarra y Novelo, 1997; Novelo, 1998); Estado de México (Bradbury, 1971); Guanajuato (García, 1997); Hidalgo (Chang, 1989).

Achnanthes minutissima var. minutissima Kützing 1833 (figuras 16)

Largo $12.2 \mu \mathrm{m}$; ancho $2.4 \mu \mathrm{m}$; estrías finas.

Datos ecológicos: perifítica, epilítica, deslizamiento, remanso.

Número de muestra: MA 51, 57, 58, 80, 90, 91, 95.

Referencias para México: México central (Metcalfe, 1985, 1988); Puebla (Avila, 1989; Ibarra, 1992; Cuesta, 1993; Ibarra y Novelo, 1997; Novelo, 1998); Guanajuato (García, 1997); San Luis Potosí (Cantoral-Uriza, 1997); Morelos (Valadez, 1998); Estado de México (Bradbury, 1971).

Cocconeis placentula Ehrenberg 1838 var. lineata (Ehrenberg 1843) Van Heurck 1880-1885 (figuras 20-21)

Largo 20.4-43.7 $\mu \mathrm{m}$; ancho 10.7-21 $\mu \mathrm{m}$; estrías $14-22$ / $10 \mu \mathrm{m}$.

Datos ecológicos: perifítica, epilítica, deslizamiento, remanso.

Número de muestra: MA 15, 25, 30, 34, 38, 42, 49, $51,55,57,58,60,61,63,64,66,80,90,93,95$.

Referencias para México: Michoacán (Hutchinson et al., 1956); Tlaxcala (Quintana, 1961); Estado de México (Bradbury, 1970, 1971; Caballero et al., 1997); México central (Kusel-Fetzmann, 1973; Metcalfe, 1985, 1988; Metcalfe et al., 1991; Metcalfe, 1992-1993); Hidalgo (Ortega, 1984; Chang, 1989); Yucatán (Sánchez, 1985); Morelos (Valadez, 1992, 1998; Valadez et al., 1996); Puebla (Navarro, 1988; Ibarra, 1992;
Cuesta, 1993; Ibarra y Novelo, 1997; Novelo, 1985, 1998; Mendoza-González, 1985); Oaxaca (Tavera y González-González, 1990); San Luis Potosí (CantoralUriza, 1990, 1993, 1997; Cantoral-Uriza y MontejanoZurita, 1993; Cantoral-Uriza et al., 1997); Guanajuato (García, 1997).

\section{Familia Naviculaceae}

* Amphora commutata Grunow, 1880 (figura 22)

En vista cingular de forma eliptica, extremos redondeados; área axial angosta, curvada, área central sólo sobre el lado ventral; rafe curveado; valvas con márgenes convexos; con extremos distales y proximales hacia el lado dorsal; estrías ligeramente radiales en toda la valva. Largo 34.3-37.9 $\mu \mathrm{m}$; ancho 5.9-6.6 $\mu \mathrm{m}$; estrías finas, centrales 12-13 / $10 \mu \mathrm{m}$ y terminales 11$12 / 10 \mu \mathrm{m}$.

Datos ecológicos: perifítica, deslizamiento.

Número de muestra: MA 37.

Referencias para México: primer registro.

Berkella linearis Ross y Sims 1978 (figura 23)

Largo 44.3-52.4 $\mu \mathrm{m}$; ancho 9.5-11 $\mu \mathrm{m}$; estrías 36-42 / $10 \mu \mathrm{m}$.

Datos ecológicos: epilítica, deslizamiento, remanso. Número de muestra: MA 80, 90, 93, 95.

Referencias para México: San Luis Potosí (CantoralUriza, 1997)

Caloneis bacillum (Grunow) Cleve 1894 (figura 24)

Largo 45.6-48.4 $\mu \mathrm{m}$; ancho 11.1-11.2 $\mu \mathrm{m}$; estrías 12$13 / 10 \mu \mathrm{m}$ centrales y terminales $15 / 10 \mu \mathrm{m}$.

Datos ecológicos: perifítica, remanso.

Número de muestra: MA 25, 58.

Referencias para México: Estado de México (Bradbury, 1971); México central (Metcalfe, 1985, 1988; Metcalfe et al., 1991); Morelos (Valadez, 1992, 1998; Valadez et al., 1996); Puebla (Ibarra, 1992; Ibarra y Novelo 1997; Cuesta, 1993; Novelo, 1998); San Luis Potosí (Cantoral-Uriza, 1997); Guanajuato (García, 1997).

Caloneis silicula (Ehrenberg) Cleve 1894 (figura 25) Largo $58.9 \mu \mathrm{m}$; ancho $13.7 \mu \mathrm{m}$; estrías 17-19 / $10 \mu \mathrm{m}$. Datos ecológicos: perifítica, deslizamiento.

Número de muestra: MA 30.

Referencias para México: Hidalgo (Ortega, 1984); Puebla (Cuesta, 1993; Novelo, 1998); Guanajuato (García, 1997).

Cymbella amphicephala Naegeli in Kützing 1849 (figura 47)

Largo $15.8 \mu \mathrm{m}$; ancho $6 \mu \mathrm{m}$; estrías dorsales 14-16 / $10 \mu \mathrm{m}$ y ventrales $17-18 / 10 \mu \mathrm{m}$. 


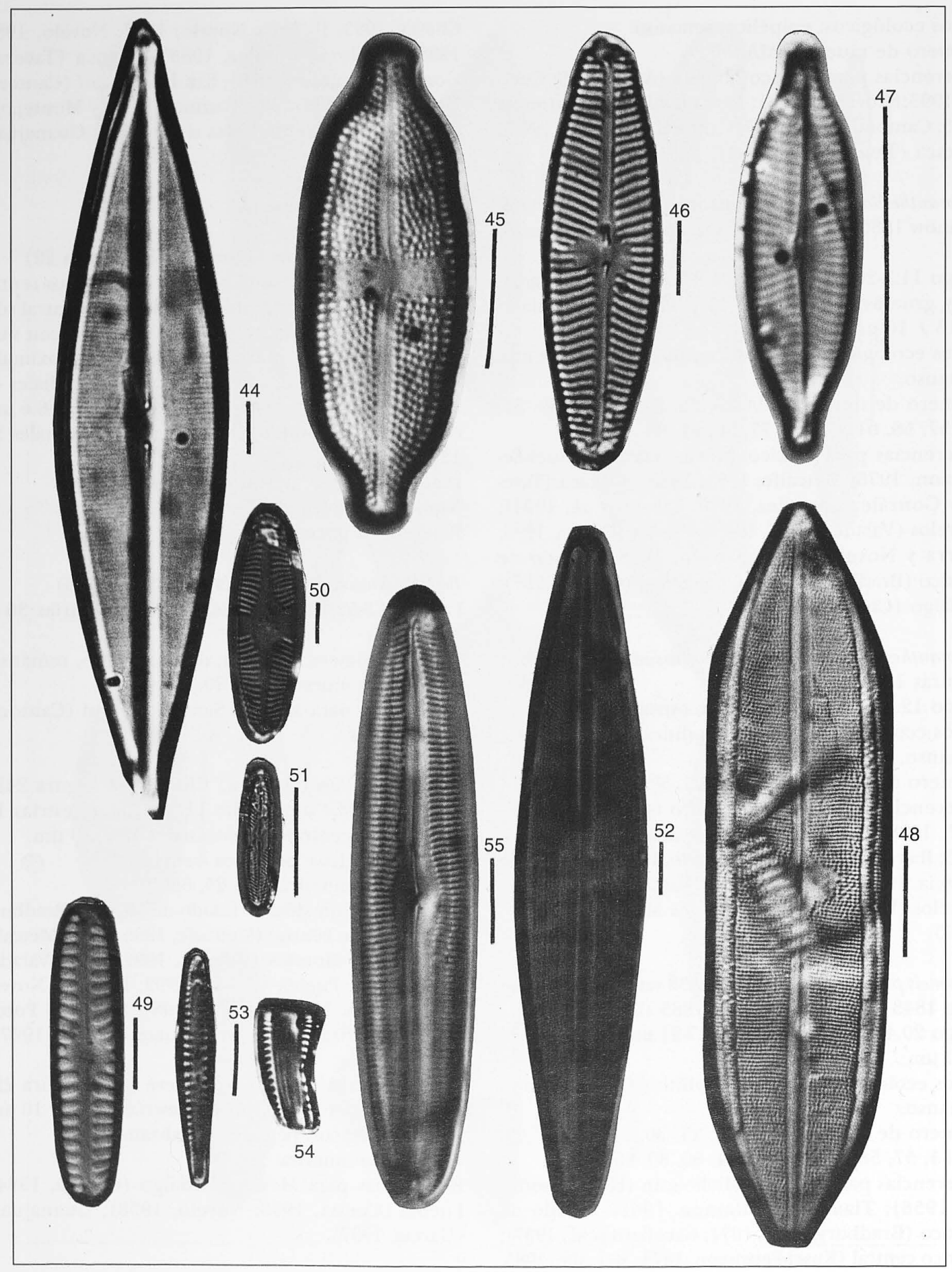

Figura 4. 44. Navicula cuspidata; 45. N. charlatii; 46. N. reinhardtii; 47. Cymbella amphicephala; 48. Neidium iridis; 49. Pinnularia borealis; 50. P. microstauron; 51. P. similis; 52. P. viridis, 53-54. Rhoicosphenia abbreviata; 55. Stauroneis phoenicenteron. Escala de la barra $10 \mu \mathrm{m}$. 
Datos ecológicos: perifítica, subaérea.

Numero de muestra: MA 58.

Referencias para México: México central (Kusel-Fetzmann, 1973); Oaxaca (Tavera et al., 1994); Puebla (Novelo, 1998).

Cymbella leptoceros (Ehrenberg) Kützing 1844 (figura 26) Largo 46.3-49.7 $\mu \mathrm{m}$; ancho 15.1-16.7 $\mu \mathrm{m}$; estrías centrales $9 / 10 \mu \mathrm{m}$ y terminales $12-13 / 10 \mu \mathrm{m}$.

Datos ecológicos: epilítica, remanso.

Numero de muestra: MA 64.

Referencias para México: Morelos (Valadez, 1992).

Cymbella mexicana (Ehrenberg) Cleve 1894 (figura 27) Largo 93.1-156.8 $\mu \mathrm{m}$; ancho 30.8-35.4 $\mu \mathrm{m}$; estrías 7-9 / $10 \mu \mathrm{m}$.

Datos ecológicos: perifítica, epilítica, epífita, deslizamiento, remanso.

Número de muestra: MA 15, 30, 37, 49, 51, 57, 61, 63. Referencias para México: Tlaxcala (Quintana, 1961); Estado de México (Ehrenberg, 1870; Bradbury, 1971); Hidalgo (Ortega, 1984); México central (Metcalfe, 1985, 1988); San Luis Potosí (Cantoral-Uriza, 1990, 1993, 1997; Cantoral-Uriza y Montejano-Zurita, 1993; Carmona, 1990, 1993; Cantoral-Uriza et al., 1997); Morelos (Valadez, 1992); Puebla (Cuesta, 1993).

Cymbella naviculiformis (Auerswald) Cleve 1894 (figura 28)

Largo 24.8-31.7 $\mu \mathrm{m}$, ancho 9.1-9.9 $\mu \mathrm{m}$; estrías centrales 13-15/10 $\mu \mathrm{m}$ y terminales $20-23 / 10 \mu \mathrm{m}$.

Datos ecológicos: epilítica, deslizamiento, remanso. Número de muestra: MA 55, 64, 90, 91, 93.

Referencias para México: San Luis Potosí (CantoralUriza, 1997).

* Cymbella norvegica Grunow in A. Schmidt et al. 1875 (figura 29)

Valvas cimbelloides con el márgen dorsal fuertemente convexo y el ventral casi recto, con una suave dilatación central, extremos anchos subrostrados a rostrados; área axial ligeramente ancha, área central ligeramente elíptica; rafe ligeramente recto, extremos proximales desplazados hacia el lado dorsal; estrías radiales en toda la valva. Largo 30.2-38 $\mu \mathrm{m}$; ancho 7.1$8.6 \mu \mathrm{m}$; estrías $12-13 / 10 \mu \mathrm{m}$.

Datos ecológicos: perifítica, deslizamiento.

Número de muestra: MA 58.

Referencias para México: primer registro.

Cymbella silesiaca Bleisch in Rabenhorst 1864 (figura 30) Largo 20.7-37.7 $\mu \mathrm{m}$; ancho 7.2-9.3 $\mu \mathrm{m}$; estrías 10-13/10 $\mu \mathrm{m}$. Datos ecológicos: perifítica, epilítica, deslizamiento, remanso.
Número de muestra: MA 30, 34, 38, 42, 49, 51, 55, $57,58,61,63,64,88,90,93,95$.

Referencias para México: San Luis Potosí (Carmona, 1990, 1993; Cantoral-Uriza y Montejano-Zurita, 1993; Cantoral-Uriza, 1997; Cantoral-Uriza et al., 1997); Oaxaca (Tavera et al., 1994); Guanajuato (García, 1997); Puebla (Novelo, 1998); Morelos (Valadez, 1998).

Cymbella sinuata Gregory 1858 (figura 31)

Largo 12.2-15.8 $\mu \mathrm{m}$; ancho 4.2-5.1 $\mu \mathrm{m}$; estrías gruesas y punteadas, ligeramente radiales, 11-12/10 $\mu \mathrm{m}$. Datos ecológicos: perifítica, epilítica, epífita, deslizamiento, remanso.

Distribución local: MA 30, 38, 51, 55, 57, 58, 63, 64, $88,90,91,93,95$.

Referencias para México: Oaxaca (Tavera et al., 1994)

Diploneis elliptica (Kützing) Cleve 1891 (figura 33)

Largo 16.7-25.6 $\mu \mathrm{m}$; ancho 9-14.3 $\mu \mathrm{m}$; estrías centrales $12-16 / 10 \mu \mathrm{m}$ y terminales $14-16 / 10 \mu \mathrm{m}$.

Datos ecológicos: perifítica, epilítica, epífita, deslizamiento, remanso.

Número de muestra: MA 30, 34, 38, 42, 49, 51, 55, 56, 57, 58, 61, 63, 64, 80, 90, 93, 95 .

Referencias para México: Estado de México (Bradbury, 1971); México central (Metcalfe, 1985, 1988); Valle de México (Flores-Granados, 1980); San Luis Potosí (Cantoral-Uriza, 1990, 1993, 1997; Cantoral-Uriza y Montejano-Zurita, 1993; Cantoral-Uriza et al., 1997); Morelos (Valadez, 1998).

Diploneis oblongella (Näegeli) Cleve-Euler 1922 (figura 32 )

Largo $30 \mu \mathrm{m}$; ancho $9.5 \mu \mathrm{m}$; estrías $18 / 10 \mu \mathrm{m}$.

Datos ecológicos: perifítica, subaérea.

Numero de muestra: MA 58.

Referencias para México: Estado de México (Bradbury, 1971); México central (Metcalfe, 1985, 1988); Guanajuato (García, 1997); San Luis Potosí (CantoralUriza, 1990, 1993, 1997; Cantoral-Uriza y MontejanoZurita, 1993).

Gomphonema acuminatum Ehrenberg 1832 (figura 36) Largo 42.3-52.1 $\mu \mathrm{m}$; ancho 7.5-17.3 $\mu \mathrm{m}$; estrías 11-12 $/ 10 \mu \mathrm{m}$

Datos ecológicos: epilítica, remanso.

Número de muestra: MA 42.

Referencias para México: México central (Kusel-Fetzmann, 1973; Metcalfe, 1985, 1988); Puebla (MendozaGonzález, 1985; Novelo, 1998); Hidalgo (Ortega, 1984).

* Gomphonema grovei M. Schmidt 1899 (figura 35)

Valva heteropolar lanceolada, estrechándose hacia los 


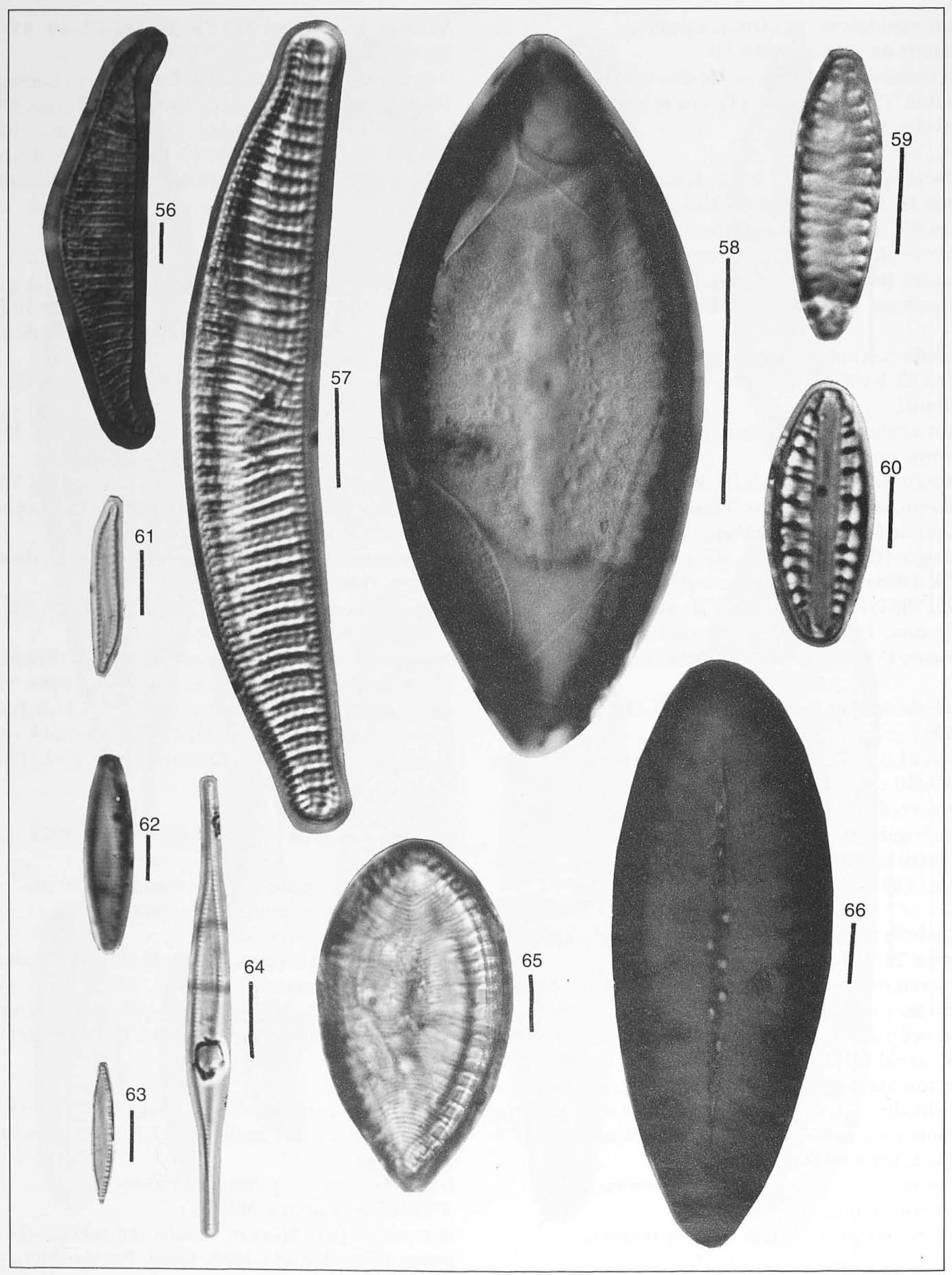

Figura 5. 56. Rhopalodia gibba; 57. Epithemia turgida; 58. Surirella bifrons (escala $50 \mu \mathrm{m}$ ); 59. S. angusta; 60. Rhopalodia gibberula; 61. Nitzschia clausii; 62. N. amphibia; 63. N. palea; 64. N. acicularis; 65. Surirella brightwelli; 66 . S. tenera. Escala de la barra $10 \mu \mathrm{m}$. 
extremos por lados cóncavos; área axial ancha, lanceolada; área central amplia, ligeramente elíptica; márgenes valvares ondulados; estrías marginales radiales en toda la valva. Largo 28.4-37.7 $\mu \mathrm{m}$; ancho 6.6$8.8 \mu \mathrm{m}$; estrías centrales $13 / 10 \mu \mathrm{m}$ y terminales $11-12$ / $10 \mu \mathrm{m}$.

Datos ecológicos: perifítica, epilítica, epífita, deslizamiento, remanso.

Número de muestra: MA 6, 7, 8,10, 11, 12, 13, 15, 20 , $25,26,27,30,31,32,33,34,35,36,37,38,39,40$, $42,43,49,50,51,52,55,57,58,61,63,64$.

Referencias para México: primer registro.

Gomphonema parvulum (Kützing) Kützing 1849 (figuras 34)

Largo $19.7 \mu \mathrm{m}$; ancho $6.2 \mu \mathrm{m}$; estrías $13 / 10 \mu \mathrm{m}$.

Datos ecológicos: perifítica, epilítica, deslizamiento, remanso.

Número de muestra: MA 51, 90, 95.

Referencias para México: Michoacán (Hutchinson $e t$ al., 1956; Metcalfe y Harrison, 1984); Estado de México (Bradbury, 1971); Valle de México (Flores-Granados, 1980); Hidalgo (Ortega, 1984; Chang, 1989); Morelos (Valadez, 1992, 1998; Valadez et al., 1996); Puebla (Navarro,1988; Ibarra, 1992; Cuesta, 1993; Ibarra y Novelo, 1997; Novelo, 1998); San Luis Potosí (Cantoral-Uriza, 1997); México central (Metcalfe, 1985, 1988; Metcalfe et al., 1991); Oaxaca (Tavera et al., 1994; Tavera y González, 1990); Guanajuato (García, 1997).

Gomphonema truncatum Ehrenberg 1832 (figuras 37) Largo $43.1 \mu \mathrm{m}$; ancho $11.4 \mu \mathrm{m}$; estrías 9-10 / $10 \mu \mathrm{m}$. Datos ecológicos: epilítica, remanso.

Número de muestra: MA 49.

Referencias para México: Hidalgo (Ortega, 1984); México central (Metcalfe, 1985); Morelos (Valadez, 1992); San Luis Potosí (Cantoral-Uriza, 1997; Cantoral-Uriza et al., 1997); Puebla (Novelo, 1998); Estado de México (Ehrenberg, 1870); Guanajuato (García, 1997).

\section{Navicula charlatii Peragallo 1921 (figura 45)}

Largo $35.4 \mu \mathrm{m}$; ancho $12.9 \mu \mathrm{m}$; estrías $17 / 10 \mu \mathrm{m}$.

Datos ecológicos: perifítica, subaérea (salpicadura)

Número de muestra: MA 58.

Referencias para México: San Luis Potosí (Carmona,1993; Cantoral-Uriza, 1997); Guanajuato (García, 1997).

Navicula cryptocephala Kützing 1844 (figura 43)

Largo 25.8-27 $\mu \mathrm{m}$; ancho 6.6-6.9 $\mu \mathrm{m}$; estrías 16-20/

$10 \mu \mathrm{m}$.

Datos ecológicos: perifítica, epilítica, deslizamiento, remanso.
Número de muestra: MA 15, 80, 95.

Referencias para México: Estado de México (Bradbury, 1970, 1971); Valle de México (Flores-Granados, 1980); Puebla (Avila, 1989; Novelo, 1985, 1998); Hidalgo (Chang, 1989); Morelos (Hernández-Becerril y TapiaPeña, 1987; Valadez, 1992); México central (Metcalfe, 1985, 1988); Guanajuato (García, 1997); San Luis Potosí (Cantoral-Uriza, 1990, 1997; Cantoral-Uriza y Montejano-Zurita, 1993).

Navicula cuspidata (Kützing) Kützing 1844 (figura 44) Largo 116.8-175.8 $\mu \mathrm{m}$; ancho 25.7-43.4 $\mu \mathrm{m}$; estrías centrales $15-16 / 10 \mu \mathrm{m}$ y terminales $20-21 / 10 \mu \mathrm{m}$.

Datos ecológicos: perifítica, epilítica, deslizamiento, remanso.

Número de muestra: MA 25, 30, 34, 37, 42, 55, 58, $61,93$.

Referencias para México: Estado de México (Bradbury, 1971); Hidalgo (Ortega, 1984); México central (Metcalfe, 1985, 1988); San Luis Potosí (Cantoral-Uriza, 1990, 1997; Cantoral-Uriza y Montejano-Zurita, 1993); Morelos (Valadez, 1992, 1998; Valadez et al., 1996); Puebla (Cuesta, 1993; Novelo, 1998); Guanajuato (García, 1997).

Navicula mutica Kützing 1844 (figura 40)

Largo $8.2 \mu \mathrm{m}$; ancho 5.3 de $\mu \mathrm{m}$; estrías $25 / 10 \mu \mathrm{m}$.

Datos ecológicos: epilítica, deslizamiento, remanso. Número de muestra: MA 80, 90.

Referencias para México: Puebla (Cuesta, 1993, Novelo, 1998); Morelos (Valadez, 1992); Michoacán (Hutchinson et al., 1956); Tlaxcala (Quintana, 1961); México central (Metcalfe, 1985, 1988; Metcalfe et al., 1991; Metcalfe, 1992-1993); Oaxaca (Tavera et al., 1994); Guanajuato (García, 1997).

\section{* Navicula nivalis Ehrenberg 1854 (figura 41)}

Valva con márgenes triondulados con extremos redondeados; área axial recta; área central amplia, con desfazamiento de estrías en ambos lados de la valva; estrías punteadas radiales en el centro, ligeramente convergentes en los extremos. Largo $23.5 \mu \mathrm{m}$; ancho $8.3 \mu \mathrm{m}$; estrías centrales $17 / 10 \mu \mathrm{m}$ y terminales $20 /$ $10 \mu \mathrm{m}$.

Datos ecológicos: perifítica, epilítica, epífita, deslizamiento, remanso.

Número de muestra: MA 57, 58, 63, 64, 93.

Referencias para México: primer registro.

Navicula pseudoscutiformis Hustedt 1930 (figura 42)

Largo 10.1-10.9 $\mu \mathrm{m}$; ancho 8.3-9.8 $\mu \mathrm{m}$; estrías 10-11/

$10 \mu \mathrm{m}$; densidad de puntos 5-6 por estría.

Datos ecológicos: perifítico, deslizamiento, remanso. Número de muestra: MA 30, 37, 51, 57. 


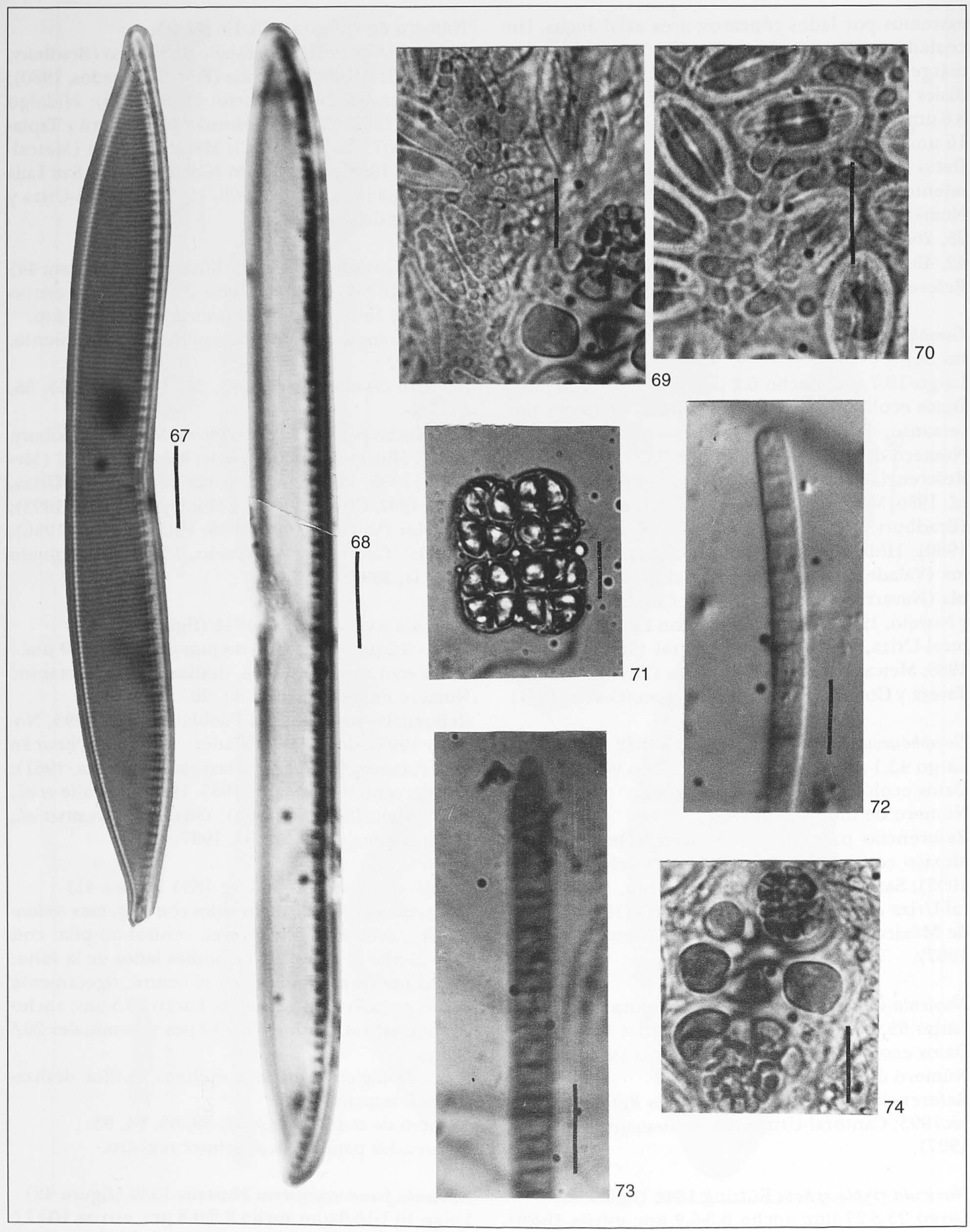

Figura 6. 67. Hantzschia amphioxys; 68. Nitzschia heufleriana; 69. Mantellum sp.; 70. Chamesiphon cf. confervicola; 71. Myxosarcina sp.; 72. Phormidium retzii; 73. Oscillatoria limosa; 74. Xenococcus cf. willei. Escala de la barra $10 \mu \mathrm{m}$. 
Referencias para México: Estado de México (KuselFetzman, 1973, Bradbury, 1971).

Navicula radiosa Kützing 1844 (figura 38)

Largo 18.3-19-9 $\mu \mathrm{m}$; ancho 4.8-4.9 $\mu \mathrm{m}$; estrías 15-19/ $10 \mu \mathrm{m}$.

Datos ecológicos: perifítica, epipélica, deslizamiento, remanso.

Número de muestra: MA 25, 34, 38, 51.

Referencias para México: Michoacán (Hutchinson et al., 1956); Estado de México (Bradbury, 1971); Hidalgo (Ortega, 1984); México central (Metcalfe, 1985, 1988); San Luis Potosí (Cantoral-Uriza, 1990, 1993, 1997; Cantoral-Uriza y Montejano-Zurita, 1993; CantoralUriza et al., 1997); Oaxaca (Tavera et al., 1994); Puebla (Novelo, 1998); Guanajuato (García, 1997).

Navicula reinhardtii (Grunow) Grunow in Cleve \& Möller 1877 (figura 46)

Largo 51.1-67.1 $\mu \mathrm{m}$; ancho 13.6-19.6 $\mu \mathrm{m}$; estrías centrales $8-7 / 10 \mu \mathrm{m}$ y terminales $8-9 / 10 \mu \mathrm{m}$.

Datos ecológicos: perifítica, epilítica, deslizamiento, remanso.

Número de muestra: MA 30, 37, 42, 55, 58, 61, 93.

Referencias para México: México central (Metcalfe, 1985).

Navicula subrhynchocephala Hustedt 1935 (figuras 39) Largo 25.1-28.7 $\mu \mathrm{m}$; ancho 6.4-7.8 $\mu \mathrm{m}$; estrías centrales $13-14 / 10 \mu \mathrm{m}$ y terminales $16-17 / 10 \mu \mathrm{m}$.

Datos ecológicos: perifítica, epilítica, epífita, epipélica, deslizamiento, remanso.

Número de muestra: MA 15, 30, 34, 37, 38, 42, 49, 51, 55, 57, 58, 61, 63, 64, 65, 66, 93.

Referencias para México: Morelos (Valadez, 1992; Valadez et al., 1996); Puebla (Cuesta, 1993; Novelo, 1998).

Neidium iridis (Ehrenberg) Cleve 1894 (figura 48)

Largo 91.4-39.4 $\mu \mathrm{m}$; ancho 23-24.8 $\mu \mathrm{m}$; estrías centrales $20-21 / 10 \mu \mathrm{m}$ y terminales $22-23 / 10 \mu \mathrm{m}$.

Datos ecológicos: perifítica, epilítica, deslizamiento, remanso.

Número de muestra: MA 25, 37, 55, 93.

Referencias para México: Estado de México (Bradbury, 1971); México central (Metcalfe, 1985).

Pinnularia borealis Ehrenberg 1843 (figura 49)

Largo $41.4 \mu \mathrm{m}$; ancho $9.8 \mu \mathrm{m}$; estrías centrales $5 / 10$ $\mu \mathrm{m}$ y terminales $6 / 10 \mu \mathrm{m}$.

Datos ecológicos: perifítica, epilítica, epipélica, deslizamiento, remanso.

Número de muestra: MA 30, 37, 55, 57, 58, 61, 63, 64, 71, 80, 93, 95 .
Referencias para México: Estado de México (Bradbury, 1971); Hidalgo (Ortega, 1984); México central (Kusel-Fetzmann, 1973; Metcalfe, 1985, 1988; Metcalfe et al., 1991); Puebla (Novelo, 1998); Guanajuato (García, 1997).

Pinnularia microstauron (Ehrenberg) Cleve 1891 (figura 50)

Largo 52.1-56.1 $\mu \mathrm{m}$; ancho 10.6-10.7 $\mu \mathrm{m}$; estrías radiales y punteadas $11-12 / 10 \mu \mathrm{m}$.

Datos ecológicos: perifítica, epilítica, deslizamiento, remanso.

Número de muestra: MA 30, 37, 55, 64, 95.

Referencias para México: Texcoco (Bradbury, 1971); Coahuila, Michoacán, Hidalgo (Ortega, 1984); México central (Metcalfe, 1985); Puebla (Novelo, 1998).

\section{Pinnularia simmilis Hustedt 1934 (figura 51)}

Largo $12.8 \mu \mathrm{m}$; ancho $4.1 \mu \mathrm{m}$; estrías 8-10/10 $\mu \mathrm{m}$.

Datos ecológicos: epilítica, epipélica, deslizamiento, remanso.

Número de muestra: MA 51, 55.

Referencias para México: Hidalgo (Ortega, 1984); México central (Metcalfe, 1985).

Pinnularia viridis (Nitzsch) Ehrenberg 1843 (figura 52) Largo $75.4 \mu \mathrm{m}$; ancho $17.8 \mu \mathrm{m}$; estrías $9 / 10 \mu \mathrm{m}$.

Datos ecológicos: perifítico, epilítico, epífito, deslizamiento, remanso.

Número de muestra: MA 37, 38, 55, 63.

Referencias para México: Hidalgo, Estado de México (Ehrenberg, 1870; Sugiura et al., 1994); Michoacán (Hutchinson et al., 1956); Hidalgo (Ortega, 1984); México central (Metcalfe, 1985, 1988; Metcalfe et al., 1991); Puebla (Cuesta, 1993; Novelo, 1998); San Luis Potosí (Cantoral-Uriza, 1997; Cantoral-Uriza et al., 1997).

Rhoicosphenia abbreviata (C. Agardh) Lange-Bertalot 1980b (figuras 53-54)

Largo 26.8-30.9 $\mu \mathrm{m}$; ancho 5.3-5.9 $\mu \mathrm{m}$; estrías centrales $11 / 10 \mu \mathrm{m}$ y terminales $14-15 / 10 \mu \mathrm{m}$.

Datos ecológicos: perifítica, epilítica, epífita, deslizamiento, remanso.

Número de muestra: MA 30, 34, 37, 38, 42, 49, 51, $55,57,58,61,63,64,72,80,90,91,93,95$.

Referencias para México: Puebla (Cuesta, 1993; Novelo, 1998); Oaxaca (Tavera et al., 1994).

Stauroneis phoenicenteron (Nitzsch) Ehrenberg 1843 (figura 55)

Largo 96.1-151.9 $\mu \mathrm{m}$; ancho 21-27.7 $\mu \mathrm{m}$; estrías centrales $13-14 / 10 \mu \mathrm{m}$ y terminales $14-15 / 10 \mu \mathrm{m}$.

Datos ecológicos: perifítica, epilítica, deslizamiento, remanso. 


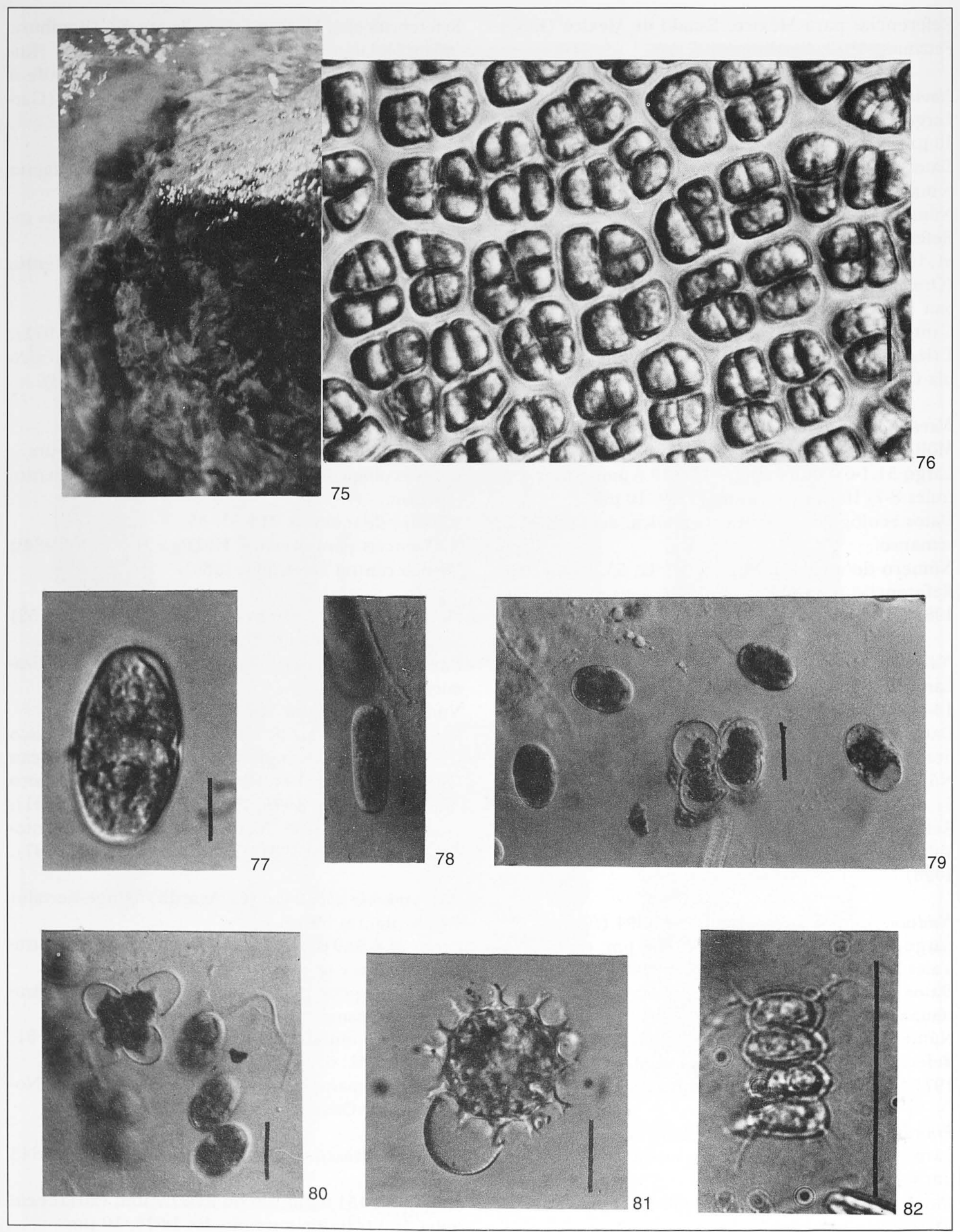

Figura 7. 75-76. Prasiola mexicana; 77-81. Penium spinospermum; 82. Scenedesmus armatus. Escala de la barra $10 \mu \mathrm{m}$. 
Número de muestra: MA 30, 34, 37, 55, 64, 90, 93, 95. Referencias para México: Estado de México (Ehrenberg, 1870: Bradbury, 1971); Hidalgo (Ortega, 1984); México central (Metcalfe, 1988); San Luis Potosí (Cantoral-Uriza, 1990, 1997; Cantoral-Uriza y Montejano-Zurita 1993).

\section{Familia Ephitemiaceae}

Epithemia turgida (Ehrenberg) Kützing 1844 var. granulata (Ehrenberg) Brun 1880 (figura 57)

Largo 42.5-115.6 $\mu \mathrm{m}$; ancho 12.1-20.8 $\mu \mathrm{m}$; costillas centrales $4 / 10 \mu \mathrm{m}$ y terminales $3-5 / 10 \mu \mathrm{m}$; estrías centrales $9-10 / 10 \mu \mathrm{m}$ y terminales de $9-10 / 10 \mu \mathrm{m}$. Estrías entre costillas centrales 3 y terminales 2 .

Datos ecológicos: perifítica, epilítica, deslizamiento, remanso.

Número de muestra: MA 15, 37, 38, 55, 57, 64, 93, 95. Referencias para México: Hidalgo (Ortega, 1984); Puebla (Novelo, 1998).

Rhopalodia gibba (Ehrenberg) O. Müller 1895 (figura 56)

Largo 93.7-96.5 $\mu \mathrm{m}$; ancho $9 \mu \mathrm{m}$; estrías $12-14 / 10 \mu \mathrm{m}$; costillas 7-8/10 $\mu \mathrm{m}$.

Datos ecológicos: perifítica, remanso.

Número de muestra: MA 34, 80.

Referencias para México: Michoacán (Hutchinson et al., 1956); Tlaxcala (Quintana, 1961); Estado de México (Bradbury, 1971); Coahuila, Michoacán (Ortega, 1984); México central (Kusel-Fetzmann, 1973; Metcalfe, 1985, 1988); Puebla (Mendoza-González, 1985; Avila, 1985, 1989; Cuesta, 1993; Novelo, 1998); Hidalgo (Chang, 1989); Oaxaca (Tavera y González-González, 1990; Tavera et al., 1994); San Luis Potosí (CantoralUriza, 1990, 1993, 1997; Cantoral-Uriza y MontejanoZurita, 1993; Carmona, 1993; Cantoral-Uriza et al., 1997); Morelos (Valadez, 1992, 1998; Valadez et al., 1996).

\section{Rhopalodia gibberula (Ehrenberg) O. Müller 1899 (fi-} gura 60)

Largo 35.7-37.1 $\mu \mathrm{m}$; ancho del frústulo 12.6-13.9 $\mu \mathrm{m}$; ancho de la valva 6.4-6.5 $\mu \mathrm{m}$; estrías $11-13 / 10 \mu \mathrm{m}$, costillas $4 / 10 \mu \mathrm{m}$; estrías entre costillas $2-4$.

Datos ecológicos: perifítica, epilítica, deslizamiento. Número de muestra: MA 30, 37, 55, 57, 58, 51.

Referencias para México: Estado de México (Bradbury, 1970); México central (Kusel-Fetzmann, 1973; Metcalfe, 1985; Metcalfe et al., 1991; Metcalfe, 1992-1993); Guanajuato y Michoacán (Metcalfe y O’hara, 1992); Hidalgo (Ortega, 1984); Morelos (Valadez, 1992, 1998; Valadez et al., 1996); Puebla (Cuesta, 1993; Ibarra y Novelo, 1997; Novelo, 1998).

\section{Familia Bacillariaceae}

Hantzschia amphioxys (Ehrenberg) Grunow in Cleve \& Grunow 1880 (figura 67)

Largo $111.4 \mu \mathrm{m}$; ancho $9.5 \mu \mathrm{m}$; estrías $15-20 / 10 \mu \mathrm{m}$; carinas $6-8 / 10 \mu \mathrm{m}$.

Datos ecológicos: perifítica, epilítica, deslizamiento, remanso.

Número de muestra: MA 15, 30, 37, 51, 55, 57.

Referencias para México: Michoacán (Hutchinson et al., 1956); Estado de México (Bradbury, 1971; Sugiura et al., 1994; Caballero et al., 1997); Hidalgo, Michoacán (Ortega, 1984); México central (Metcalfe, 1985, 1988; Metcalfe et al., 1991; Metcalfe, 1992-1993); Puebla (Novelo, 1985, 1998; Navarro, 1988; Avila, 1989); Oaxaca (Tavera et al., 1994); Guanajuato (García, 1997).

Nitzschia acicularis (Kützing) W. Smith 1853 (figura 64) Largo $52.5 \mu \mathrm{m}$; ancho $4 \mu \mathrm{m}$; fíbulas $16 / 10 \mu \mathrm{m}$.

Datos ecológicos: perifítica, remanso.

Número de muestra: MA 66.

Referencias para México: Puebla (Avila, 1989; Novelo, 1998); Morelos (Valadez, 1998); México central (Metcalfe, 1985, 1988).

Nitzschia amphibia Grunow 1862 (figura 62)

Largo $40.5 \mu \mathrm{m}$; ancho $6.3 \mu \mathrm{m}$; estrías $30 / 10 \mu \mathrm{m}$; fíbulas $15 / 10 \mu \mathrm{m}$.

Datos ecológicos: perifítica, epilítica, deslizamiento, remanso.

Número de muestra: MA 34, 51, 55, 57, 61, 64, 66. Referencias para México: Michoacán (Hutchinson et al., 1956); Estado de México (Bradbury, 1970, 1971; Caballero et al., 1997); Yucatán (Sánchez, 1985); México central (Metcalfe, 1985, 1988; Metcalfe et al., 1991; Metcalfe, 1992-1993); Puebla (Avila, 1985, 1989; Navarro, 1988; Ibarra, 1992; Cuesta, 1993; Ibarra y Novelo,1997; Novelo, 1985, 1998); Oaxaca (Tavera y González-González, 1990; Tavera et al., 1994); San Luis Potosí (Cantoral-Uriza, 1990, 1997; Cantoral-Uriza y Montejano-Zurita 1993); Morelos (Valadez, 1992, 1998; Valadez et al., 1996); Guanajuato (García, 1997); Hidalgo (Ortega, 1984).

Nitzschia clausii Hantzsch 1860 (figura 61) Largo 25.1-25.3 $\mu \mathrm{m}$; ancho 3.9-4.3 $\mu \mathrm{m}$; fíbulas 9-13/ $10 \mu \mathrm{m}$.

Datos ecológicos: epipélica, remanso.

Número de muestra: MA 64, 80.

Referencias para México: Morelos (Valadez, 1992; Valadez et al., 1996); San Luis Potosí (Cantoral-Uriza, 1997); Puebla (Navarro, 1988; Avila, 1989; Cuesta, 1993; Novelo, 1998); México central (Metcalfe, 
1985, 1988); Guanajuato (García, 1997); Estado de México (Bradbury, 1971).

Nitzschia heufleriana Grunow 1862 (figura 68)

Largo 105.6-138.7 $\mu \mathrm{m}$; ancho 3.1-6.2 $\mu \mathrm{m}$; fíbulas 9-13/ $10 \mu \mathrm{m}$.

Datos ecológicos: perifítica, epilítica, epipélica, deslizamiento, remanso.

Número de muestra: MA 25, 34, 38, 51, 55, 57, 64, 80, 90, 93, 95 .

Referencias para México: Morelos (Valadez, 1992, 1998; Valadez et al., 1996); Puebla (Ibarra, 1992).

Nitzschia palea (Kützing) W. Smith 1856 (figura 63) Largo 14.9-25.3 $\mu \mathrm{m}$; ancho 2.9-4.1 $\mu \mathrm{m}$; fíbulas 12-15/ $10 \mu \mathrm{m}$.

Datos ecológicos: perifítica, epilítica, epipélica, deslizamiento, remanso.

Número de muestra: MA 34, 51, 55, 58, 61, 80, 91, 93. Referencias para México: Estado de México (Bradbury, 1971); México central (Metcalfe, 1985, 1988; Metcalfe et al., 1991; Metcalfe, 1992-1993); Guanajuato y Michoacán (Metcalfe y O’hara, 1992); Morelos (Hernández-Becerril y Tapia-Peña, 1987); Puebla (Navarro, 1988; Avila, 1989; Ibarra, 1992; Cuesta, 1993; Ibarra y Novelo, 1997; Novelo, 1998); San Luis Potosí (Cantoral-Uriza 1997); Guanajuato (García, 1997); Hidalgo (Chang, 1989).

\section{Familia Surirellaceae}

Surivella angusta Kützing 1844 (figura 59)

Largo $25.9 \mu \mathrm{m}$; ancho $7-2 \mu \mathrm{m}$ de ancho; fíbulas $7 /$ $10 \mu \mathrm{m}$.

Datos ecológicos: perifítica, deslizamiento.

Número de muestra: MA 37.

Referencias para México: Puebla (Novelo, 1998); Oaxaca (Tavera et al., 1994). Estado de México (Bradbury, 1971).

Surireila bifrons Ehrenberg 1843 (figura 58)

Largo 204.1-215.7 $\mu \mathrm{m}$; ancho 74.8-86.2 $\mu \mathrm{m}$; fíbulas 16$17 / 10 \mu \mathrm{m}$

Datos ecológicos: perifítica, remanso.

Numero de muestra: MA 25.

Referencias para México: Coahuila, Guanajuato, Hidalgo (Ortega, 1984); San Luis Potosí (Cantoral-Uriza, 1997).

* Surirella brightwellii W. Smith 1853 (figura 65) Valva heteropolar ancha, lanceolada, extremos ligeramente redondeados; área axial indistinguible. Largo $59.9 \mu \mathrm{m}$; ancho $18.9 \mu \mathrm{m}$; fíbulas $3 / 10 \mu \mathrm{m}$.

Datos ecológicos: epipélica, remanso.
Número de muestra: MA 64.

Referencias para México: primer registro.

Surirella tenera Gregory 1856 (figura 66)

Largo 85.5-96.8 $\mu \mathrm{m}$; ancho 38-40.1 $\mu \mathrm{m}$, fíbulas $2 / 10 \mu \mathrm{m}$. Datos ecológicos: perifítica, epilítica, deslizamiento, remanso.

Número de muestra: MA 25, 34, 55, 64, 93.

Referencias para México: Michoacán (Hutchinson et al., 1956); Estado de México (Bradbury, 1971); México central (Kusel-Fetzmann, 1973; Metcalfe, 1985, 1988); Puebla (Navarro, 1988; Avila, 1989; Cuesta, 1993; Novelo, 1998); Morelos (Valadez, 1992, 1998; Valadez et al., 1996); Guanajuato (García, 1997); San Luis Potosí (Cantoral-Uriza, 1997).

\section{Clase Cyanoprokariota}

Orden Chroococcales

Familia Merismopediaceae

Subfamilia Merismopedioideae

Mantellum Dangeard 1941 sp. (figura 69)

Células esféricas a ligeramente ovales arreglados en un plano, solitarias o en grupos, de 2.2-3.9 $\mu \mathrm{m}$ de diámetro. Contenido celular homogéneo.

Datos ecológicos: perifítica, epífita y sobre plástico, remanso.

Número de muestra: MA 52, 54.

Referencia para México: nivel de género.

\section{Familia Xenococcaceae}

\section{Myxorsarcina Printz $1921 \mathrm{sp}$. (figura 71)}

Colonias de $25 \mu \mathrm{m}$ de diámetro, con mas de 32 células. Paquetes celulares envueltos por una vaina delgada, firme e hialina, de 1-1.5 $\mu \mathrm{m}$. Células negras o gris azul-verdoso, contenido homogéneo, de 4.5-6.5 $\mu \mathrm{m}$ de diámetro. Baeocitos cubiertos raramente, de 4-8 en número.

Se han citado dos especies para este género en Europa, una es marina y otra para ambientes continentales, alrededor de 8 especies citadas fuera de Europa (India, USA, Sudáfrica, Indonesia, Puerto Rico); pero ninguna coincide con el conjunto de carateres, por lo que decidimos no asignarle hasta el momento un epíteto específico.

Datos ecológicos: perifítica, epilítica, epífita, subaérea.

Número de muestras: MA 49

Referencias para México: nivel de género.

Xenococcus cf. willei Gardner 1927 (figura 74)

Células sésiles, más o menos hemisféricas, en agregados que crecen en una capa paralela al sustrato. 
Células con contenido celular homogéneo o finamente granuloso. Vaina incolora. Las células se dividen por fisión binaria o por la formación de nanocitos. Células de 3.9-10.5 $\mu \mathrm{m}$ de diámetro, 4.9-13.0 $\mu \mathrm{m}$ de largo; nanocitos 2.0-4.1 $\mu \mathrm{m}$ de diámetro.

Datos ecológicos: perifítica, epífita y sobre plástico, deslizamiento.

Número de muestra: MA 5, 52.

Referencias para México: México central (Montejano et al., 1993; Gold-Morgan et al., 1994).

\section{Familia Chamaesiphonaceae}

Chamaesiphon cf. confervicolus A. Braun in Rabenhorst 1865 (figura 70 )

Filamentos claviformes, células de 2 a $2.5 \mu \mathrm{m}$ de diámetro, 3 a $5 \mu \mathrm{m}$ de largo. Contenido celular homogéneo; vaina hialina, delgada. Uno o dos nanocitos por célula.

Datos ecológicos: perifítica, epífita y sobre plástico, deslizamiento.

Número de muestra: MA 5, 52.

Referencias para México: San Luis Potosí (Montejano et al., 1993; Valadez et al, 1996).

Orden Oscillatoriales

Familia Phormidiaceae

Subfamilia Phormidioideae

Phormidium retzii (C. Agardh) Kützing 1892 (figura 72) Matas de filamentos de color negro. Filamentos de 3.7-4.1 $\mu \mathrm{m}$ de diámetro, tricomas con vaina. Células de 2.1-3.7 $\mu \mathrm{m}$ de largo, contenido celular granuloso. Célula apical redondeada.

Referencias ambientales: perifítica, epilítica, remanso; crecimiento visible.

Número de muestra: MA 14.

Referencias para México: Morelos (Valadez, 1992, 1998; Valadez et al., 1996); Puebla (Ávila, 1985).

\section{Subfamilia Oscillatorioideae}

Oscillatoria limosa C. Agardh 1892 (figura 73)

Filamentos de 4.5-5.6 $\mu \mathrm{m}$ de diámetro y células de 1.8$3.8 \mu \mathrm{m}$ de largo, contenido celular granuloso, con gránulos evidentes en los septos. Célula apical redondeada a ligeramente aguzada.

Referencias ambientales: perifítica, epilítica, remanso; crecimiento visible.

Número de muestra: MA 14.

Referencias para México: Morelos (Valadez et al., 1996); Valle de México (Flores-Granados, 1980; Sámano-Bishop, 1933); Oaxaca (Sámano-Bishop, 1948); Puebla (Ávila, 1985; Novelo, 1998).
Clase Chlorophyceae Orden Chlorellales

Familia Scenedesmaceae

Scenedesmus armatus Chod, (figura 82)

Cenobios de 12.5-17.5 mm de largo, 3.5-11.5 mm de ancho; células de 3.5-4.6 $\mu \mathrm{m}$ de diámetro, con un pirenoide evidente por célula. Células de los extremos con espinas en ambos polos. Pared celular con una costilla estriada.

Datos ecológicos: perifítica, epipélica, estanque.

Número de muestra: MA 81.

Referencias para México: Morelos (Valadez, 1992, 1998; Valadez et al., 1996).

\section{Clase Ulvophyceae}

Orden Ulvales

Familia Prasiolaceae

Prasiola mexicana J. Agardh 1867 (figuras 75-76)

Talos laminares distromáticos de color verde obscuro, de hasta $15 \mathrm{~cm}$ de altura. Superficie lisa o corrugada. Células dispuestas en tétradas y margen firme. Datos ecológicos: perifítica, epilítica, deslizamiento y rara en remansos, descle la interfase hasta $50 \mathrm{~cm}$ de profundidad; crecimiento visible.

Número de muestras: MA 1, 3, 4, 9, 12, 26, 27, 29, $36,39,43,45,49,56$.

Referencias para México: Veracruz (Ortega, 1984).

Clase Charophyceae

Orden Zygnematales-Desmideales

Familia Mesotaeniaceae

* Penium spinospermum Joshua 1883 (figuras 77-81) Células elípticas o cilíndricas con una ligera constricción media y ápices redondeados, $25.5-42.9 \mathrm{~mm}$ de largo, 18.7-31.8 mm de ancho. Pared estriada, hileras longitudinales punteadas consistentes. Cloroplasto axial con un pirenoide central. Zigosporas esféricas o cúbicas de $22.6-37.5 \mathrm{~mm}$ de diámetro incluídas las proyecciones; cubiertas con espinas que en el extremo se bifurcan.

Datos ecológicos: perifítica, epipélica, subaérea; crecimiento visible.

Número de muestra: MA 49.

Referencias para México: primer registro.

\section{Discusión}

La flora algal del río La Magdalena estuvo representada principalmente por especies de la clase Bacillariophyceae, este grupo es de forma frecuente el que mayor número de especies presenta en estos ambien- 
tes. La representante de Ulvophyceae, Prasiola mexicana fue el taxa que formó talos macroscópicos visibles en las dos épocas del año. El porcentaje de especies nuevas $15.3 \%$ es importante, no es raro en una zona y país que han sido poco estudiados y por ello este número seguramente se incrementará.

Se presentó una variación en la diversidad de taxa entre localidades la cual, puede estar explicada por las diferentes épocas del año; siendo la temperatura del agua y la velocidad de corriente las variables que presentaron mayor fluctuación. Asimismo, las localidades con mayor diversidad y que más especies compartieron fue el cuarto y segundo dinamo, sitios que se encuentran protegidos de la zona urbana.

La composición de especies descrita en el presente trabajo difiere de las floras registradas en otros ambientes lóticos del país (Montejano et al., 2000; Valadez et al., 1996) y tiene un cierto parecido con ríos de regiones templadas, de aguas frías o de alta montaña (Suxena y Venkateswarlu, 1967; Whitton y Dalpra, 1967; Parker et al., 1973); cabe resaltar la presencia de especies en el río La Magdalena en las condiciones antes mencionadas a Cymbella norvegica, Navicula pseudoscutiformis, Pinnularia borealis y Prasiola mexicana. Algunas comunidades algales encontradas en el río La Magdalena corresponden a la flora de corrientes de montaña encontradas en México (Ortega, 1984); podemos citar a especies de corrientes como Diatoma mesodon, Meridion circulare var. constrictum y Nitzschia heufleriana.

Los 11 nuevos registros para el país reflejan la necesidad de estuaiiar los afluentes del Valle de México y trabajar intensivamente la biología de algunas especies. Para los géneros que no se pudieron determinar a especie, se requieren realizar cultivos para conocer diferentes fases de sus historias de vida y obtener estructuras reproductoras, que son caracteres fundamentales, tal es el caso de las Charophyceae, Ulvophyceae, Chlorophyceae y Xanthophyceae, mientras que para Cyanophyceae se requiere observar más material. A la par de ir resolviendo los problemas taxonómicos, es necesario una aproximación al estudio de las comunidades algales que nos permita entender su estructurra y dinámica desde un punto de vista ecológico.

\section{Agradecimientos}

A la M. en C. Silvia Espinosa por su asesoría en el microscopio electrónico de barrido, al Dr. Gustavo Montejano por su ayuda en la determinación de las Cyanophyceae y a la Dra. Marina Aboal por la revisión y comentarios del manuscrito. Proyecto financiado por el programa CONSERVA 1998 de la Secretaría del
Medio Ambiente del Gobierno del Distrito Federal.

\section{Literatura citada}

Anagnostidis K. y Komárek J. 1985. Modern approach to the classification system of Cyanophytes. 1. Introduction. Archiv für Hydrobiologie / Supplement 71, Algological Studies 38-39:291-302.

Anagnostidis K. y Komárek J. 1988. Modern approach to the classification system of Cyanophytes. 3. Oscillatoriales. Archiv für Hydrobiologie / Supplement 80, Algological Studies 50-53:327-472.

Avila N.J. 1985. Ficoflora manifiesta del suelo del Valle de Tehuacán, Puebla. México. Tesis de licenciatura, Facultad de Ciencias, UNAM, México, 147 pp.

Avila N.J. 1989. Ficoflora potencial de suelo húmedo del Valle de Tehuacán, Puebla. Tesis de maestría, Facultad de Ciencias, UnAM, México, 93 pp.

Bradbury J.P. 1970. Diatoms from the Pleistocene sediments of Lake Texcoco, Mexico. Rev. Géogr. Phys. Géol. Dynam. 12(2):161-168.

Bradbury J.P. 1971. Paleolimnology of lake Texcoco, Mexico. Evidence from Diatoms. Limnology and Oceanography 16(2):180-200.

Caballero M., Macías J.L., Lozano-García S. y Urrutia-Fucugauchi J. 1997. Late Pleistocene-Holocene volcanic stratigraphy and paleoenvironments of the upper Lerma basin, Mexico. Special volume on lacustrine sedimentation.

Caballero M. y Ortega B.1998. Lake levels since about 40,000 years ago at Lake Chalco, near Mexico City. Quaternary Research 50: 69-70.

Cantoral-Uriza E.A. 1990. Ficoflora de ambientes lénticos en la localidad El Salto, en la Huasteca Potosina. Tesis de licenciatura, Facultad de Ciencias, UNAM, México, 139 pp.

Cantoral-Uriza E.A. 1993. Ecología de comunidades algales del sistema hidrológico El Salto, en la Huasteca Potosina. Tesis de maestría, Facultad de Ciencias, UNAM, México, $97 \mathrm{pp}$.

Cantoral-Uriza E.A. 1997. Diatomeas (Bacillariophyceae) de ambientes lóticos en la cuenca baja de la Huasteca Potosina. México. Tesis doctoral, Facultad de Ciencias, UNAM, México, 201 pp.

Cantoral-Uriza E.A., Carmona J.J. y Montejano G. 1997. Diatoms of calcareous tropical springs in the central region of Mexico. Cryptogamie Algologie 18(1):19-46.

Cantoral-Uriza E.A. y Montejano G. 1993. Las algas de la localidad "El Salto" (San Luis Potosí, México): un ejemplo de estudios florísticos en ambientes cambiantes. Boletín de la Sociedad Botánica de México 53:3-20.

Carmona J.J. 1990. Ficoflora de manantiales de la Huasteca Potosina, México. Tesis de licenciatura, Facultad de Ciencias, UNAM, México, 105 pp. 
Carmona J.J. 1993. Taxonomía del género Audouinella (Acrochaetiales: Rhodophyta) en la cuenca baja del río Pánuco, México. Tesis de maestría, Facultad de Ciencias, UNAM, México, 90 pp.

Cuesta Z.I. 1993. Ecología de la ficoflora de los Manantiales de San Lorenzo, Tehuacán, Puebla. Tesis de licenciatura, Facultad de Ciencias, UNAM, México, 119 pp.

Chang W.L.A. 1989. Variación de la comunidad planctónica de la Presa Requena, Hidalgo. México, Tesis de licenciatura, Facultad de Ciencias, UNAM. México, 112 pp.

Desikachary T.V. 1959. Cyanophyta. Indian Council of Agricultural Research Institute. New Delhi. 686 pp.

Ehrenberg C.G. 1870. Aus mikroskopischen Bacillarien Unter und ei der studt Mexiko: Berlin. Buchdruckerei der Königl Akademie der Wissenschaften. Berlin. 66 pp.

Espinosa G.F. y Sarukhán J. 1997. Manual de malezas del Valle de México. Universidad Nacional Autónoma de MéxicoFondo de Cultura Económica. México. 407 pp.

Figueroa T.G. 1984. Estudio ecológico de la ficoflora de la presa Miguel Alemán, Oaxaca. México. Tesis de licenciatura, Facultad de Ciencias, UNAM, México, 120 pp.

Flores-Granados C. 1980. Variaciones estacionales en la composición florística del fitoplancton de dos canales de Xochimilco, D.F. México. Tesis de licenciatura, Escuela Superior de Ciencias Biológicas, IPN, México, 139 pp.

García M.J.V. 1997. Diatomeas de una zona de jales mineros de Guanajuato. Tesis de licenciatura, Facultad de Ciencias, UNAM, México, 87 pp.

Geitler L. 1930-1932. Cyanophyceae. L. Rabenhorst's Ed. Kryptogamen-Flora von Deutschland, Österreich und der Schweiz. Akademische Verlagsgesellschaft m.b.h. Leipzig. $1196 \mathrm{pp}$.

Germain H. 1981. Flore des diatomées. Diatomphycées, eaux douces et saumatres du Massif Armoricain et des contrées voisines d'Europe occidentale. Société Nouvelle des éditions Boubée. Paris. 444 pp.

Gold-Morgan M., Montejano G. y Komárek J. 1994. Freshwater epiphytic Cyanoprocaryotes from central Mexico. Archiv für Protistenkunde 144: 383-405.

Gold-Morgan M., Montejano G. y Komárek J. 1996. Freshwater epiphytic Chamaesiphonaceae from central Mexico. Algological Studies 83:257-271.

Hernández-Becerril D.U. y Tapia M.I. 1987. Ecología del fitopláncton primaveral de superficie en el lago de Tequesquitengo, Morelos, México. Revista de Biología Tropical 35(1):31-39.

Hock C. v.d., Mann D y Jahns H.M. 1995. Algae: an introduction to Phycology. Trans. by D. Mann. Germany-USA. Koeltz Scientific Books. 576 pp.

Hustedt F. 1927-1930. Die Kieselalgen Deutschlands, Österreichs und der Angrenzenden Meeresgebiete. En: Rabenhorst's L. Eds., Kryptogamen-Flora von Deutschland, Österreich und der Schweiz. 7, Teil 1. First reprinting, 1971. Strauss y Cramer GmbH, Leutershausen. 920 pp.

Hustedt F. 1930. Bacillariophyta (Diatomeae). En: Pascher A. Ed., Die Susswasser-Flora Mitteleuropas, Heft. 10. Verlag Von Gustav Fischer, Jena. 467 pp.

Hustedt F. 1931-1959. Die Kieselalgen Deutschlands, Österreichs und der Schweiz mit Berücksichtigung der Ubrigen Läuder Europas souie der Angrenzenden Meeresgebiete. En: Rabenhorst's L. Eds. Kryptogamen-Flora von Deutschland, Österreich und der Schweiz. 7, Teil 2. First reprinting, 1971. Strauss y Cramer GmbH, Leutershausen. 845 pp.

Hutchinson G.E., Patrick R. y Deevey E.S. 1956. Sediments of Lake Pátzcuaro, Michoacán, México. Bulletin of the Geological Society of America 67:1491-1504.

Ibarra V.C. 1992. Flora Diatomológica de Texcala, Tehuacán, Puebla. Tesis de licenciatura, Facultad de Ciencias, UNAM, México, 80 pp.

Ibarra V.C. y Novelo E. 1997. Diatomeas de Texcala, Puebla. Boletín de la Sociedad Botánica de México 61:49-57.

Instituto Nacional de Estadística Geografía e Informática. 1995. Cuaderno Estadístico Delegacional. Magdalena Contreras, D.F., México.

Komárek J. y Anagnostidis K. 1986. Modern approach to the classification system of cyanophytes. 2. Chroococcales. Archiv für Hydrobiologie / Supplement 73, Algological Studies 43:157-226.

Komárek J. y Anagnostidis K. 1989. Modern approach to the classification system of cyanophytes. 4. Nostocales. Archiv für Hydrobiologie / Supplement 82, Algological Studies 56:247-345.

Komárek J. y Anagnostidis K. 1999. Cyanoprokaryota. Teil 1: Chroococcales. Gustav Fischer. Germany.

Krammer K. y Lange-Bertalot H. 1986. Bacillariophyceae. Teil 1: Naviculaceae. Band 2/1. Die Sübwasserflora von Mitteleuropa, Begr. Pascher, A.; Ettl, H., Gerloff J., Heynig H. y Mollenhauer D. Eds., Gustav Fischer Verlag, Sttutgart. 876 pp.

Krammer K. y Lange-Bertalot H. 1988. Bacillariophyceae. Teil 2: Bacillariaceae, Ephitemiaceae, Surirellaceae. Band 2/2. Die Susswasserflora von Mitteleuropa, Begr. Pascher, A.; Ettl H., Gerloff J., Heynig H. y Mollenhauer D. Eds., Gustav Fischer Verlag, Sttutgart. 596 pp.

Krammer K. y Lange-Bertalot H. 1991a. Bacillariophyceae. Teil 3. Centrales, Fragilariaceae, Eunotiaceae. Band 2/3. Die Susswasserflora von Mitteleuropa. Begr. Pascher, A.; Ettl H., Gerloff J., Heynig H. y Mollenhauer D. Eds., Gustav Fischer Verlag, Sttutgart. 576 pp.

Krammer K. y Lange Bertalot H. 1991b. Bacillariophyceae Teil 4. Achnanthaceae, Kritische Ergänzungen zu Navicula (Lineolatae) und Gomphonema Gesamtliteraturverzeichnis Teil 14. Die Susswasserflora von Mitteleuropa. Begr. Pascher, A.; Ettl, H., Gärtner G., Gerloff J., Heynig H. y Mollenhauer D. Eds., Gustav Fischer Verlag, Sttutgart. 437 pp.

Kusel-Fetzmann E. 1973. Beitrage zur Kenntnis der Algen- 
flora der Hochgebirge Zentralamerikas und der nördlichen Anden. Hochgebirgsforschung 3:29-68.

Lowe R.L. 1974. Environmental requirements and pollution tolerance of freshwater diatoms. National environmental research center and development. Environmental protection agency. Cincinnati, Ohio. USA. 334 pp.

Mendoza-González C. 1985. Estudio florístico ficológico estacional de la Laguna Victoria o de Santiago Tilapa, México. Phytologia 58(7):479-487.

Metcalfe S.E. 1985. Late Quaternary environments of central Mexico: A diatom record. $\mathrm{Ph}$. D. Diss. Hertford College and the School of Geography. Oxford, USA.

Metcalfe S.E. 1988. Modern diatom asemmblages in central Mexico: the role of water chemistry and other environmental factors as indicated by TWISPAN and DECORANA. Freshwater biology 19:217-33.

Metcalfe S.E. y Harrison S.P. 1984. Cambio ambiental del cuaternario tardío en depósitos lacustres en la cuenca de Zacapu, Michoacán, reconstrucción preliminar. Bol. Inst. Geografía Méx. 14: 127-151.

Metcalfe S.E., Street-Perrot F.A., Perrot R.A. y Harkness D.D. 1991. Paleolimnology of the Upper-Lerma Basin, Central Mexico: A record of climatic change and anthropogenic disturbance since 11,600 yr BP. Journal of Paleolimnology 5: 197-218.

Metcalfe S.E. y O'hara S.L. 1992. Sensibilidad de lagos mexicanos a alteraciones en el medio ambiente: ejemplos del Eje Neovolcánico. Ingeniería Hidráulica en México: 107-121.

Metcalfe S.E. 1992-1993. Changing environments of the Zacapu basin, central Mexico: A diatom-based history spanning the last 30,000 years. The Editors, Research papers, School of Geography, Oxford.

Montejano G., Gold-Morgan M. y Komárek J. 1993. Freshwater epiphytic cyanoprocaryotes from central Mexico. I. Cyanocystis \& Xenococcus. Archiv für Protistenkunde 143:237-247.

Montejano G., Gold-Morgan M. y Komárek J. 1997. Freshwater epiphytic cyanoprocaryotes from central Mexico. III. The genus Stischosiphon Geitler 1932. Archiv für Protistenkunde 148:3-16.

Montejano Z.G., Carmona-Jiménez J. y Cantoral-Uriza E. 2000. Algal communities from calcareous springs and streams in La Huasteca, central Mexico: a synthesis. En: Munawar, M., Lawrence S.G., Munawar I.F. y Malley D.F. Eds. Aquatic Ecosystems of Mexico: Status \& Scope. Backhuys Publishers, Leiden, 135-149.

Navarro J.L.E. 1988. Un estudio tónico de Rhoicosphenia curvata (Kütz.) Grunow ex Rabenhorst var. curvata en el Valle de Tehuacán, Puebla. Tesis de licenciatura, Facultad de Ciencias, UNAM, México, 120 pp.

Novelo M.E. 1985. Flora dinámica del suelo del Valle de Tehuacán, Puebla. México. Tesis de maestría, Facultad de Ciencias, UNAM, México, 151 pp.
Novelo M.E. 1998. Floras ficológicas del valle de Tehuacán, Puebla. Tesis doctoral, Facultad de Ciencias, UNAM, México, 599 pp.

Ortega M.M. 1984. Catálogo de algas continentales recientes de México. UnAM. México. 566 pp

Parker B.C., Samsel G.L. y Prescott G.W. 1973. Comparison of microhabitats of microscopie subalpine stream algae. The american Midland Naturalist 90:143-153.

Patrick R. y Reimer C.W. 1966. The Diatoms of the United States. No. 13. Vol. I. Monographs of the Academy of Natural Sciences of Philadelphia. Pennsylvania. 688 pp.

Patrick R. y Reimer C.W. 1975. The Diatoms of the United States. No. 13. Vol. II. Monographs of the Academy of Natural Sciences of Philadelphia. Pennsylvania. 213 pp.

Prescott G.W., Croasdale H.T. y Vinyard W.C. 1975. A synopsis of North american desmids. Part II. Desmidiaceae: Placodermae. Section 1. Lincoln, University of Nebraska Press, USA, $275 \mathrm{pp}$.

Quintana G.G. 1961. Estudio de las diatomeas fósiles de México en el microscopio electrónico y en el espectrofotómetro de rayos X. Tesis de licenciatura, Facultad de Ciencias, UnAM, México, $79 \mathrm{pp}$.

Round F.E. 1984. The Ecology of algae. Cambridge University Press. Great Britain. $653 \mathrm{pp}$.

Rushforth S.R., Kaczmarska I. y Johansen J.R. 1984. The Subaerial Diatom Flora of Thurston Lava Tube, Hawaii. Bacillaria, 7:135-157.

Sámano-Bishop A. y Sokoloff D. 1931. La flora y fauna de aguas dulces del Valle de México. Monogr. Inst. Biol. Univ. Nac. México 1: 48-49.

Sámano-Bishop A. 1933. Algunas cianoficeas del lago de Xochimilco. Anales Inst. Biol. Univ. Nal. Autón. México 4:29-31.

Sámano-Bishop A. 1948. Observaciones preliminares de la flora algológica de la región de Tuxtepec, Oaxaca. An. Inst. Biol. Mex. 19(2):317-333.

Sánchez M.I. 1985. Flora planctónica de los cenotes de Yucatán. Bacillariophyta. Mérida, Yucatán. Tesis de licenciatura, Universidad Autónoma de Yucatán, México. 90 pp.

Sheath R. y Cole K.M. 1992. Biogeography of stream macroalgae in North America. Journal of Phycology 28(4):448460.

Sugiura Y., Flores A., Ludlow B., Valadez C.F., Gold-Morgan M. y Maillol J. 1994. El agua, la tierra, el bosque y el hombre en el Alto Lerma: un estudio multidisciplinario. Resultados preliminares. Arqueologia 11-12:29-45.

Suxena M.R. y Venkateswarlu V. 1967. Algae of the Cho Oyn (E. Himalaya) Expedition-I Bacillariophyceae. Hidrobiologia 32:

Tavera S.R.L. y González-González J. 1990. Caracterización ficoflorística de los paredones de la Sierra de Juárez, Oaxaca. Importancia de las formas de crecimiento algales en la tipificación de un ambiente. Boletín de la Sociedad Botánica de México 50: 121-133. 
Tavera S.R.L., Elster J. y Marvan P. 1994. Diatoms from Papaloapan basin communities, Mexico. Archiv für $\mathrm{Hy}$ drobiologie / Supplement 104, Algological Studies 74:35-65.

Transeau N.E. 1951. The Zygnemataceae. Fresh-water conjugate algae. The Ohio State University Press. Columbus. 327 pp.

Valadez C.F. 1992. Flora ficológica de ambientes lóticos de la cuenca baja del Río Amacuzac, Morelos. Tesis de licenciatura, Facultad de Ciencias, UNAM, México, 239 pp.

Valadez C.F. 1998. Empleo de crecimientos algales para la caracterización de ambientes lóticos en la cuenca baja del río Amacuzac, Morelos. Tesis de maestría, Facultad de Ciencias, UNAM, México, 116 pp.

Valadez C.F., Carmona J.J. y Cantoral-Uriza E.A. 1996. Algas de ambientes lóticos en el Estado de Morelos, México. Anales del Instituto de Biologia, Serie Botánica 67(2):227282.

Ventakatamaran G.S. 1961. Vaucheriaceae. Indian council of Agricultural Research Institute. New Delhi. 112.

Whitton B. A. y Dalpra M. 1967. Floristic Changes in the River Tees. Hidrobiologia 32:545-550. 